\title{
Propagation of curved shock fronts using shock ray theory and comparison with other theories
}

\author{
By S. BASKAR AND PHOOLAN PRASAD ${ }^{2} \dagger$ \\ ${ }^{1}$ Department of Mathematics, Indian Institute of Science, Bangalore-560012, India \\ ${ }^{2}$ Centre for Plasma Astrophysics, Department of Mathematics, Kathdick University, Leuven, \\ 3001 Leuven, Belgium
}

(Received 13 October 2003 and in revised form 2 September 2004)

Shock ray theory (SRT) has been found to be useful and computationally efficient in finding successive positions of a curved weak shock front. In this paper, we solve some piston problems and show that the shock ray theory with two compatibility conditions gives shock positions, which are very close to those obtained by solving the same problems by the numerical solution of Euler's equations (Euler solutions). Comparison of the results obtained by shock ray theory and geometrical shock dynamics (GSD) of Whitham (J. Fluid Mech. vol. 2, 1957, p. 146) with the Euler solution shows that the shock ray theory gives more accurate results for any piston motion. The aim of the work is not just this comparison, but also to investigate the role of the nonlinearity in accelerating the process of the evolution of a shock, produced by an explosion of a non-circular finite charge, into a circular shock front. We find that the nonlinear waves propagating on the shock front appreciably accelerate this process. We also discuss a situation, for shock Mach number very close to 1 , when GSD and shock ray theory may fail to give any result.

\section{Introduction}

A blast wave produced by the explosion of a charge of finite size will initially have a non-spherical shape, or rather non-circular shape for this paper since we consider here only the propagation of a cylindrical shock described in the $(x, y)$-plane. The initial shape will be non-circular, not only because the explosive may be packed in a non-circular container, but also because not all parts of the explosion would burn simultaneously. After a long time, when the leading shock has travelled a large distance compared to the linear dimensions of the explosion, the shock front will be almost circular even according to the linear theory. However, nonlinearity present in Euler's equations of motion (we consider propagation of the shock front in a polytropic gas) will tend to smoothen the geometry of the shock front owing to nonlinear waves propagating on the shock front itself and the shock front may become almost circular much earlier. We investigate this phenomenon by the shock ray theory. Prasad (1993) first derived the shock ray theory for a weak shock directly from the transport equations along a shock ray for a shock of arbitrary strength and later Monica \& Prasad (2001) derived it from a weakly nonlinear ray theory (see Prasad 2001 for all references and a detailed discussion). The weakly nonlinear

$\dagger$ Permanent address: Department of Mathematics, Indian Institute of Science, Bangalore-12, India. prasad@math.iisc.ernet.in. 
ray theory (Prasad 2000) is a WKB theory, generalized to a hyperbolic system of quasi-linear equations. The shock ray theory is ideally suited for this investigation since:

(i) It provides the shock as a well-defined sharp curve in computational results.

(ii) It requires very small computational time to give successive positions of the shock. In fact, only a fraction of the time required for computing the shock position by the numerical solution of Euler's equations called the Euler solution in this paper.

(iii) It gives a critical time $t_{c}$, an estimate of the time when the shock curve is very nearly a circle.

(iv) It gives results very close to those obtained by solving Euler's equations.

Shock ray theory consists of shock ray equations (Prasad 1982) and an infinite system of compatibility conditions along these rays (Grinfel'd 1978; Maslov 1980). However, the system of equations for successive compatibility conditions becomes too complex to be of any use. Suitable truncation of these equations in the $n$th compatibility condition leads to a finite system of equations (Ravindran \& Prasad 1990), which simplify considerably for a weak shock (Prasad 1993; Monica \& Prasad 2001). When we refer to the shock ray theory in this paper, we mean the ray equations with two compatibility conditions and with suitable truncation in the second compatibility condition for a weak shock. Kevlahan (1996) provided evidence for the property (iv) for shock ray theory by comparing its results with some known exact solutions, with experimental results and some numerical solutions of Euler's equations. Since, Kevlahan did not have the conservation form of the equations of shock ray theory, his comparison with Euler's results is only for a limited time. In this paper, we show that there is an excellent agreement of the results of shock ray theory with the Euler solution through an extensive numerical computation, even for those cases in which there is some doubt as to the validity of shock ray theory, i.e. when the curved piston is accelerating.

In $\S 2$, we give a new formulation of the conservation forms of the two compatibility conditions used in shock ray theory here. These conservation forms appear to be more natural and follow a pattern, which is valid for each of the infinite set of compatibility conditions for a curved shock of an arbitrary strength. In $\S 3$, we derive the initial condition to set up the initial-value problem for the system of equations of the shock ray theory appropriate to the flow produced by the motion of a curved piston. In subsequent sections, we present the results of three problems solved by shock ray theory and compare the results of shock ray theory, the Euler solution and the results of geometrical shock dynamics (GSD) by Whitham (1957).

The presence of weakly nonlinear ray theory results provides an excellent opportunity for comparison of the results. In the theory of a weak shock, all important features of the shock arise over the distance between the linear wavefront and the shock. This is why we study weak shock governed by $u_{t}+\left(a_{0}+\epsilon u\right) u_{x}=0$, where $\epsilon$ is a small quantity, by the model equation $u_{t^{\prime}}+u u_{x^{\prime}}=0$ with $t^{\prime}=t, x^{\prime}=\left(x-a_{0} t\right) / \epsilon$. From a general theorem (Prasad 2001, theorem 9.2.1, p. 267), it follows that this distance, which is the displacement (measured suitably for a multi-dimensional shock) of a weak shock from the linear wavefront, is of the same order as the displacement of the nonlinear wavefront (by weakly nonlinear ray theory) from the weak shock. The nonlinear wavefront has the same topological shape as the shock. Therefore, an error in the position of the GSD (and shock ray theory) is to be calculated in terms of the ratio of the distance (appropriately defined) of the GSD shock (or shock ray theory shock) from the Euler solution shock, to the distance of the GSD shock (or shock ray theory shock) from the nonlinear wavefront. Another quantity, which is important 
for comparison, is the shock strength, which has been measured by Monica $\&$ Prasad (2001). The GSD shock, while propagating in a uniform medium, does not decay (amplify), but shock ray theory shock does, as when waves from behind catch up and modify the shock strength for a decelerating (accelerating) piston problem.

Since no estimation of the error of the shock ray theory seems to be possible, specially for curved shocks, and the theory is very important for applications (for example, from focused sonic boom in aviation (Plotkin 2002) to shock wave lithotripsy to treat kidney stone disease (Sturtevant 1989)), it is important to compare the results of shock ray theory with Euler's results. This has become more important because the GSD have been used to predict finer results of the shape of shock fronts in some limiting cases (Apazidis et al. 2002; Schwendeman 2002). Even if a theory has only $5 \%$ error, the limiting results may be completely wrong. Hence our comparison of the results by shock ray theory, GSD and Euler's equations are valuable. This comparison becomes more important for us because we wish to answer the question raised in the beginning of this section, 'How does the nonlinearity present in Euler's equations accelerate the process of a non-circular shock to become circular?' The answer is provided by shock ray theory at the end of the $\S 6$ and, therefore, we must examine the reliability of the results of shock ray theory. We find excellent agreement between the results of shock ray theory and Euler's results, whereas those between GSD and Euler's results are not as good. We also discuss some limitations in the application of weakly nonlinear ray theory, GSD and shock ray theory in solving a piston problem with a convex corner in the piston.

\section{Conservation form of the equations of shock ray theory}

Let us consider a cylindrical shock propagating into a polytropic gas at rest and in a uniform state $(\rho, \boldsymbol{q}, p)=\left(\rho_{0}, \mathbf{0}, p_{0}\right)$, where $\rho$ is the density, $\boldsymbol{q}=\left(q_{1}, q_{2}\right)$ the velocity and $p$ is the pressure. Let $a$ be the sound velocity in the medium: $a^{2}=\gamma p / \rho$, where $\gamma$ is the ratio of specific heats. Propagation of such a shock can be studied in the $(x, y)$-plane. We assume the shock to be produced by the motion of a curved piston. Before proceeding further, we introduce a non-dimensional coordinate system with the help of a length $L$ and the sound velocity $a_{0}$ in the uniform state. We choose $L$ to be a length of the order of the linear dimension of the piston. We denote the non-dimensional coordinates also by the same symbol $(x, y, t)$. The basic equations from which the shock ray theory has been derived are the well-known Euler equations.

We assume the piston to be at rest for $t<0$ and then start moving suddenly with a small non-zero velocity at $t=0$ and with a small acceleration. This produces a shock front initially coincident with the piston. The shock will be followed by a oneparameter family of nonlinear wavefronts which are also the result of the piston motion. However, these nonlinear wavefronts are identifiable over only a small distance behind the shock, because the high-frequency (or the short-wave) approximation is valid only over such a distance from the shock. It also follows that the unit normal to the shock front and that of any one of the nonlinear wavefronts behind it are approximately the same.

Let $N=(\cos \Theta, \sin \Theta)$ be the unit normal of the shock front. Assuming the shock to be weak, the perturbation in density $\rho$, fluid velocity $\boldsymbol{q}$ and pressure $p$ up to a short distance behind the shock are given by (see Prasad 2001, chap. 10)

$$
\rho-\rho_{0}=\rho_{0} w, \quad \boldsymbol{q}=N a_{0} w, \quad p-p_{0}=\rho_{0} a_{0}^{2} w,
$$


where $w$ is of the order of a small quantity $\epsilon$, which is a measure of the shock strength. We denote the value of $w / \epsilon$ on the shock front by $\mu$ and the Mach number of the shock by $M$, and they are given by

$$
\mu=\left.(w / \epsilon)\right|_{\text {shock front }}, \quad M=1+\epsilon \frac{\gamma+1}{4} \mu .
$$

Under a short-wave assumption $\langle\boldsymbol{N}, \nabla\rangle w$ is assumed to be of order 1 . We now define a quantity $V$ by

$$
V=\left.\frac{\gamma+1}{4}\{\langle N, \nabla\rangle w\}\right|_{\text {shock front }} .
$$

Note that this quantity was earlier denoted by $N$ (Monica \& Prasad 2001; Prasad 2001) and is of $O(1)$.

We introduce a ray coordinate system $(\xi, t)$ such that $\xi=$ constant are shock rays in the $(x, y)$-plane and $t=$ constant are successive positions of the shock. Let $G$ be the metric associated with $\xi$ (see Prasad 2001, for the definition), and $(X, Y)$ be a point on the shock at time $t$. The equations of shock ray theory for a weak shock are (Monica \& Prasad 2001; Prasad 2001, these references may be consulted for a detailed explanation of all concepts mentioned briefly here)

$$
\begin{aligned}
& X_{t}=M \cos \Theta, \quad Y_{t}=M \sin \Theta, \\
& \Theta_{t}+\frac{1}{G} M_{\xi}=0, \quad G_{t}-M \Theta_{\xi}=0, \\
& M_{t}+\frac{M-1}{2 G} \Theta_{\xi}+(M-1) V=0, \quad V_{t}+\frac{V}{2 G} \Theta_{\xi}+2 V^{2}=0 .
\end{aligned}
$$

If we eliminate $\Theta_{\xi}$ between $(2.5 b)$ and $(2.6 a)$, we obtain

$$
\frac{2 M}{M-1} M_{t}+\frac{G_{t}}{G}+2 V M=0 .
$$

In order to discuss shocks in the solutions of system (2.5)-(2.6), in the ( $\xi, t)$-plane, we require the system to be in conservation form. Two physically realistic conservation laws, called kinematical conservation laws (KCL), representing conservation of distance in two independent directions and equivalent to (2.5) for differentiable functions $M, \Theta$ and $G$, are

$$
(G \sin \Theta)_{t}+(M \cos \Theta)_{\xi}=0, \quad(G \cos \Theta)_{t}-(M \sin \Theta)_{\xi}=0 .
$$

We derive now two new conservation forms, which not only follow a general pattern valid for all compatibility conditions, but are particular cases for a shock of arbitrary strength (Prasad 2004). We notice in (2.6) for the shock strength $M-1$ and the gradient $V$ behind the shock that the second terms have a coefficient $\Theta_{\xi} /(2 G)$, which represents geometric amplification of decay of the corresponding quantities $M-1$ and $V$, respectively. To derive a conservation form of the equations involving $M-1$, we take the (2.7), which gives a combination $\left\{F^{\prime}(h) / F(h)\right\} h_{t}+G_{t} / G$ where $h=M-1$ and $F$ is a known function of $h$, or more specifically $F(h)=h^{2} \mathrm{e}^{2 h}$, leading to the conservation form

$$
\left\{G(M-1)^{2} \mathrm{e}^{2(M-1)}\right\}_{t}+2 M(M-1)^{2} \mathrm{e}^{2(M-1)} G V=0 .
$$

Similarly, eliminating $\Theta_{\xi}$ between $(2.5 b)$ and (2.6b), we obtain an equation which we rewrite as

$$
V_{t}+\frac{V}{2 G} G_{t}+\frac{V}{2}\left(\frac{1}{M}-1\right) \frac{G_{t}}{G}+2 V^{2}=0 .
$$


We use (2.7) to replace $G_{t} / G$ in the third term by $-2 M M_{t} /(M-1)-2 V M$ (note $M_{t}=$ $\left.(M-1)_{t}\right)$, which gives the conservation form

$$
\left\{G V^{2} \mathrm{e}^{2(M-1)}\right\}_{t}+G V^{3}(M+1) \mathrm{e}^{2(M-1)}=0 .
$$

The conservation forms (2.9) and (2.10) of the compatibility conditions (2.6a) and $(2.6 b)$, respectively, appear to be physically realistic and are different from those used by Monica $\&$ Prasad (2001). In the linear theory, the energy conservation along a ray tube is represented by $\left\{G(M-1)^{2}\right\}_{t}=0$, which can be written in an integral formulation using two cross-sections of a ray tube (see equation (7.69) and the next equation in Whitham 1974). Nonlinearity seems to bring in a factor $\mathrm{e}^{2(M-1)}$, as seen in Prasad (2001, chap. 4 on weakly nonlinear ray theory; see also Prasad \& Sangeeta 1999) and the dissipation of energy through a shock is represented by the source terms in (2.9) and (2.10). Any other form containing an expression $\{f(G F(h))\}_{t}$, where $f: \mathbb{R} \rightarrow \mathbb{R}$ is a monotonic function, also appears to give appropriate geometrical decay or amplification of $h$. In this case, a jump relation across a shock is given by $G_{r} F\left(h_{r}\right)=$ $G_{l} F\left(h_{l}\right) \Leftrightarrow f\left(G_{r} F\left(h_{r}\right)\right)=f\left(G_{l} F\left(h_{l}\right)\right)$, where $l$ and $r$ represent the states on the two sides of a shock.

The system of four equations (2.5)-(2.6) is hyperbolic for $M>1$, which is true for a shock. Thus, we obtain a system of four equations in conservation form: (2.8)-(2.10), which is hyperbolic for a shock front. Given a solution of this system, we solve (2.4) as ordinary differential equations for each value of $\xi:(X=X(\xi, t), Y=Y(\xi, t))$, which give the position of the shock front at a fixed time $t$ and a ray for a fixed $\xi$. Given an initial position of a shock $\Omega_{0}:\left(X_{0}(\xi), Y_{0}(\xi)\right.$ ) (so that we can compute $\Theta_{0}(\xi)$ ), initial shock strength $M_{0}(\xi)$ and initial gradient $V_{0}(\xi)$ of the flow behind $\Omega_{0}$; we can set up an initial-value problem of shock ray theory equations (2.4) and (2.8)-(2.10)

$X(\xi, 0)=X_{0}(\xi), \quad Y(\xi, 0)=Y_{0}(\xi), \quad \Theta(\xi, 0)=\Theta_{0}(\xi), \quad M(\xi, 0)=M_{0}(\xi), \quad V(\xi, 0)=V_{0}(\xi)$.

The problem of finding successive positions of a shock front is reduced from a threedimensional problem of solving Euler's equations in $(x, y, t)$-space to that of solving the shock ray theory equations in the $(\xi, t)$-plane. This reduction of one dimension leads to a considerable computational saving. In fact, in the problems we have solved in this paper, shock ray theory takes less than $10 \%$ of the time required for solving Euler's equations. Moreover, since we must solve a system of hyperbolic equations in conservation form, we can use highly sophisticated and powerful numerical schemes. In this work, we used the discontinuous Galerkin finite-element method (Cockburn, San \& Shu 1989) for solving the hyperbolic system. Further, we have used a sourceterm-splitting method for the inhomogeneous terms appearing in shock ray theory and a Strang-dimension-splitting method for solving a two-dimensional Euler system. In the next section, we determine the initial values for the shock ray theory equations for a shock front produced by the impulsive motion of a curved piston.

\section{Initial conditions for shock ray theory equations for a piston problem}

Consider now the disturbance produced by the impulsive motion of a piston. For a small time, the distance between the shock and the piston is small compared to the extent of the piston. Thus, in the initial stages, the entire flow produced by the piston satisfies the high-frequency or the short-wave approximation (this is in contrast to the situation for a large time, when only the flow immediately behind the shock satisfies this approximation). For a small time, we can now visualize the flow between the piston and the shock as being generated by a one-parameter family of nonlinear 
wavefronts. For such a small time, we take the unit normal $\boldsymbol{n}_{p}$ of the piston to be equal to those of the nonlinear wavefronts $\boldsymbol{n}$ and the shock $\boldsymbol{N}$ (i.e. $\boldsymbol{n}_{p}=\boldsymbol{n}=\boldsymbol{N}$ ). A moving curve is associated with a ray coordinate system $(\xi, t)$ of its own. In principle, we can choose the ray coordinate system of the piston to be different from that of any one of the nonlinear wavefronts and that of the shock. We can do this in spite of a condition we impose that in the limit as $t$ tends to zero, the variable $\xi$ appearing in these is the same as $\xi$ of the piston, which we can choose (at $t=0)$ to be the arclength along the piston. However, in order to derive the initial conditions for the shock ray theory equations, we shall equate the $\xi$-derivatives along all these curves and hence we must choose the ray coordinate system of the piston to be such that $\xi$ is not only the arclength along the initial position of the piston, but $\xi=$ constant and $t=$ constant curves form an orthogonal system in the $(x, y)$-plane, as in the case of the shock front and a nonlinear wavefront. This choice means that if the piston surface is represented by

$$
(x, y)=\left(x_{p}(\xi, t), y_{p}(\xi, t)\right)
$$

then

$$
\boldsymbol{n}_{p}=\boldsymbol{x}_{p t} /\left|\boldsymbol{x}_{p t}\right|
$$

Let the equation of the shock be represented by $(x, y)=(X(\xi, t), Y(\xi, t))$, then

$$
X_{0}(\xi)=X(\xi, 0)=x_{p}(\xi, 0), \quad Y_{0}(\xi)=Y(\xi, 0)=y_{p}(\xi, 0) .
$$

Therefore, we can calculate $\Theta_{0}(\xi)$ from the initial shape of the piston. Now, we proceed to calculate $M_{0}(\xi)$ and $V_{0}(\xi)$. We note that the boundary condition at the piston in an inviscid flow is given by the fluid speed on the piston in the normal direction is equal to the piston speed in the normal direction. This gives

$$
w\left(\boldsymbol{x}_{p}(\xi, t), t\right) \equiv\left\langle\boldsymbol{n}_{p}(\xi, t), \boldsymbol{q}\left(\boldsymbol{x}_{p}(\xi, t), t\right)\right\rangle=\left\langle\boldsymbol{n}_{p}(\xi, t), \boldsymbol{x}_{p t}(\xi, t)\right\rangle=\left|\boldsymbol{x}_{p t}\right| .
$$

From (2.2) and (3.4), we obtain

$$
M_{0}(\xi)=1+\frac{\gamma+1}{4}\left|\boldsymbol{x}_{p t}\right| .
$$

The transport equation for the amplitude $w$ (from weakly nonlinear ray theory, equation (10.1.4), Prasad 2001) takes the form

$$
w_{t}+\left(1+\frac{\gamma+1}{2} w\right)\langle N, \nabla\rangle w=\Omega w
$$

where $\Omega=-\langle\nabla, N\rangle / 2$ is the mean curvature of a nonlinear wavefront behind the shock front in the short-wave limit. Taking its limit as we approach the piston, we obtain, after using (3.4),

$$
\left.w_{t}\right|_{p}+\left.\left(1+\frac{\gamma+1}{2}\left|\boldsymbol{x}_{p t}\right|\right)\left\langle\boldsymbol{n}_{p}(\xi, t), \nabla\right\rangle w\right|_{p}=\left.(\Omega w)\right|_{p} .
$$

We shall now encounter two types of partial derivatives with respect to $t$, one when $\boldsymbol{x}$ is kept fixed and another when $\xi$ is kept fixed. The result, (3.4), is valid for all $t>0$ and taking its derivative with respect to $t$, we obtain

$$
\left\{\left.w_{t}\right|_{p}+\left.\left(\left\langle\boldsymbol{x}_{p t}, \nabla\right\rangle w\right)\right|_{p}\right\}=\left\langle\boldsymbol{x}_{p t}, \boldsymbol{x}_{p t t}\right\rangle /\left|\boldsymbol{x}_{p t}\right|=\left\langle\boldsymbol{n}_{p}, \boldsymbol{x}_{p t t}\right\rangle,
$$

which at $t=0$, after using (3.2), becomes

$$
\left.w_{t}\right|_{p, t=0}+\left.\left|\boldsymbol{x}_{p t}(\xi, 0)\right|\left\langle\boldsymbol{n}_{p 0}, \nabla\right\rangle w\right|_{p, t=0}=\left.\left\langle\boldsymbol{n}_{p}, \boldsymbol{x}_{p t t}\right\rangle\right|_{t=0} .
$$


Setting $t=0$ in (3.7) and eliminating $\left.w_{t}\right|_{p, t=0}$ between (3.7) and (3.8), we obtain (we note $\left.\Omega\right|_{t=0}=$ mean curvature of the piston at $t=0$ is equal to $\left.\Omega_{p}\right|_{t=0}$ and use $\boldsymbol{n}_{p 0}$ for $\left.\boldsymbol{n}_{p}(\xi, 0)\right)$

$$
\begin{aligned}
\left\{\left(1+\left.\frac{\gamma+1}{2} w\right|_{p, t=0}\right)-\left|\boldsymbol{x}_{p t}(\xi, 0)\right|\right\} & \left\{\left.\left\langle\boldsymbol{n}_{p 0}, \nabla\right\rangle w\right|_{p, t=0}\right\} \\
& =\left(\left.\left.\Omega_{p}\right|_{t=0} w\right|_{p, t=0}\right)-\left\langle\boldsymbol{n}_{p 0}, \boldsymbol{x}_{p t t}(\xi, 0)\right\rangle .
\end{aligned}
$$

We now use (3.4) in (3.9) and note (2.3) to derive the initial value $V(\xi, 0)=V_{0}(\xi)$ as

$$
V_{0}(\xi)=\frac{\gamma+1}{4\left\{1+\frac{1}{2}(\gamma-1)\left\langle\boldsymbol{n}_{p 0}, \boldsymbol{x}_{p t}(\xi, 0)\right\rangle\right\}}\left[\left.\Omega_{p}\right|_{t=0}\left|\boldsymbol{x}_{p t}(\xi, 0)\right|-\left\langle\boldsymbol{n}_{p 0}, \boldsymbol{x}_{p t t}(\xi, 0)\right\rangle\right] .
$$

Note that the values $M_{0}(\xi)$ and $V_{0}(\xi)$ are completely determined by (3.5) and (3.10) in terms of the initial shape and initial motion of the curved piston. The initial value $G_{0}=G(\xi, 0)$ is obtained from the initial geometry of the shock front, which is the same as that of the piston. Hence,

$$
G_{0}(\xi)=\left|\boldsymbol{x}_{p \xi}(\xi, 0)\right| .
$$

In this paper, we shall use very simple geometrical forms of the piston, which will be either a symmetrically expanding square or a curve made of a number of straight segments and moving as a rigid line in the direction of a symmetry. Then, $\boldsymbol{n}_{p 0}$ is piecewise constant, i.e. $\Omega_{p}=0$ except for a set $S$ of isolated points and also $\boldsymbol{n}_{p t}=0$ except for $S$. In this case, the expression for $V_{0}(\xi)$ simplifies considerably to

$$
V_{0}(\xi)=-\frac{\gamma+1}{4\left\{1+\frac{1}{2}(\gamma-1)\left|\boldsymbol{x}_{p t}\right|\right\}}\left\langle\boldsymbol{n}_{p 0}(\xi), \boldsymbol{x}_{p t t}(\xi, 0)\right\rangle .
$$

Note that in $\boldsymbol{x}_{p t}$, the time derivative of $\boldsymbol{x}_{p}$ is with $\xi=$ constant, i.e. $\left|\boldsymbol{x}_{p t}\right|$ is the normal speed of the piston.

\section{Other theories}

In this section, we shall describe other theories, with which we shall compare the results of shock ray theory. The simplest of these is the linear theory. This is a wellknown theory, in which rays in a uniform medium are straight lines and the wavefront is given by Huygens' method. It is also well known that in the linear theory, the ray method from a smooth part of the initial wavefront gives the same wavefront as the Huygens' method, but the ray method fails near singularities of the initial wavefront.

\subsection{Weakly nonlinear ray theory}

When we consider a shock front behind which the flow satisfies the high-frequency or short-wavelength approximation, the shock front is followed by a one-parameter family of nonlinear waves. These waves, when weak, follow the weak shock front, catch up with the shock, interact and then disappear from the flow. The evolution of any one of these wavefronts is also governed by the kinematical conservation laws (see Prasad 2001),

$$
(g(m) \sin \theta)_{t}+(m \cos \theta)_{\xi}=0, \quad(g(m) \cos \theta)_{t}-(m \sin \theta)_{\xi}=0,
$$

where $m$ is the Mach number of the weakly nonlinear wavefront, $\theta$ the angle between the normal to the wavefront and the $x$-axis, and the metric $g$ associated with the 
coordinate $\xi$ is given by

$$
m=1+\frac{1}{2}(\gamma+1) w, \quad g(m)=(m-1)^{-2} \mathrm{e}^{-2(m-1)},
$$

provided the variable $\xi$ is chosen suitably.

The system (4.1) is hyperbolic if $m>1$ and (2.1) implies that the pressure $p$ on the wavefront is greater than that in the ambient medium. We only consider the case when $m>1$. Once we have a solution $m=m(\xi, t), \theta=\theta(\xi, t)$ of (4.1), we can find the position of the wavefront by solving

$$
x_{t}=m \cos \theta, \quad y_{t}=m \sin \theta .
$$

The system of equations (4.1) and the ray equations (4.3) forms the weakly nonlinear ray theory and gives the complete history of weakly nonlinear waves which are continuously produced by the piston. A weakly nonlinear wave, which instantaneously coincides with the shock front, heading the disturbance in the piston problem, is produced by the piston, not at the time $t=0$ when the shock is produced, but at a later time. However, we compare the history of a nonlinear wavefront with that of the shock because the evolution of both are topologically and qualitatively the same, and the geometrical shock dynamics (GSD) is almost the same as the weakly nonlinear ray theory except that the relation for $m$ in (4.2) is replaced by (2.2). In this paper, we shall discuss only one nonlinear wavefront, the one that was produced by the piston at the same time as the shock was produced; but this is done only as an academic exercise because it is annihilated by the shock as soon as it is produced. We calculate only its geometry and position without worrying that it does not exist. The initial condition for $\theta$ for this weakly nonlinear wavefront are the same as those for $\Theta$ in the $\S 3$ (i.e. $\theta(\xi, 0)=\theta_{p}$ ) and that for $m$ is $m(\xi, 0)=1+(\gamma+1) /\left.2 w\right|_{p}=m_{0}$, say.

\subsection{Whitham's geometrical shock dynamics}

The kinematical conservation laws (2.8) (or (4.1)) govern the evolution of any moving curve in a plane. The additional closure equations such as (2.9) and (2.10) in shock ray theory or (4.2) for weakly nonlinear ray theory come from the dynamics of the curve. Whitham did not have the kinematical conservation laws, but derived its differential form (2.5), and then, using his valuable insight into the physics of the problem, provided a closure relation (now well known as the $\mathrm{A}-\mathrm{M}$ relation), which for a weak shock becomes

$$
G(M)=(M-1)^{-2},
$$

provided we again choose the variable $\xi$ suitably. Note that for a weak shock, the ray tube area $A \propto(M-1)^{-2}$. The two relations (4.2) and (4.4) agree up to the first term in the expansion of the right-hand side of (4.2) for small $m-1$. Whitham's intuition, which led to (4.4), clearly shows the self-propagation property, a property characteristic of weakly nonlinear wavefronts (Prasad 1995; also see Prasad 2001), but was used for a shock front. By GSD, we mean here not the differential form of equations by Whitham, but kinematical conservation laws (2.8) along with (2.4) and (4.4). One of our main aims in this paper is to compare the results of shock ray theory and GSD with the Euler solution.

\subsection{Euler's equations of motion}

The conservation form of the Euler equations of motion of a polytropic gas are well known. We have already commented on non-dimensionalization of space and time coordinates in $\S 2$. We include that and additional non-dimensional variables 
(with a prime)

$$
\rho^{\prime}=\rho / \rho_{0}, \quad \boldsymbol{q}^{\prime}=\boldsymbol{q} / a_{0}, \quad p^{\prime}=p /\left(\gamma p_{0}\right), \quad \boldsymbol{x}^{\prime}=\boldsymbol{x} / L, t^{\prime}=a_{0} t / L,
$$

and then drop the prime from the non-dimensional variables in the transformed equations. The non-dimensional form of the Euler equations remain same as the original equations.

The equilibrium state ahead of the shock (or the nonlinear wavefront) is $\left(\rho_{0}, \boldsymbol{q}_{0}, p_{0}\right)=(1,0,1 / \gamma)$, so that the perturbation (2.1) becomes

$$
\rho=1+w, \quad \boldsymbol{q}=\boldsymbol{N} w, \quad p=\frac{1}{\gamma}+w .
$$

The shock and nonlinear wavefront Mach numbers are given by (2.2) and (4.2), respectively. Given the piston motion and its geometry in the form (3.1), we can use (3.5) and (3.10) (or (3.12)) to set up the initial-value problem for the shock ray theory; (3.5) alone for GSD and

$$
m(\xi, 0)=1+\frac{\gamma+1}{2}\left|\boldsymbol{x}_{p t}\right|
$$

for weakly nonlinear ray theory. Equation (4.7) follows from (3.4) and (4.2).

Before we close this section, we discuss a superficial relation between the weakly nonlinear ray theory and shock ray theory. As discussed by Whitham (1959), the solution of an initial-value problem of (2.8)-(2.10) for small time $t$ tend to the solution with the same initial values of (2.8) and

$$
\left\{G(M-1)^{2} \mathrm{e}^{2(M-1)}\right\}_{t}=0, \quad\left\{G V^{2} \mathrm{e}^{2(M-1)}\right\}_{t}=0 .
$$

With a proper choice of $\xi$, i.e. the initial value of the metric $G,(4.8 a)$ gives $G=$ $(M-1)^{-2} \mathrm{e}^{-2(M-1)}$. Therefore, it appears that for a small time, the shock ray theory shock is governed by the equations of the weakly nonlinear ray theory, but this is only a superficial relation because it is the difference in the initial values in (3.5) for $M_{0}(\xi)$ and (4.7) for $m_{0}(\xi)$ which makes a nonlinear wavefront and a shock front be distinct propagating curves. If we approximate $(M-1)^{-2} \mathrm{e}^{-2(M-1)}$ by $(M-1)^{-2}$ for small $M-1$, then it follows that initially, near the source of creation of the shock, the shock ray theory shock is governed approximately by GSD equations.

\section{Piston problem when the shape of the piston is a wedge}

Consider a wedge-shaped piston which starts moving with velocity $u_{0}+u_{1} t$ in the direction of the symmetry, assumed to be the direction of the $x$-axis. Let $\xi$ be the distance along the piston measured from the vertex. Then for $t>0$ and $u_{0}>0$,

$$
x_{p}(\xi, t)=\left\{\begin{array}{ll}
-\xi \sin \Theta_{0}+\left(u_{0} t+\frac{1}{2} u_{1} t^{2}\right), & \xi>0, \\
\xi \sin \Theta_{0}+u_{0} t+\frac{1}{2} u_{t} t^{2}, & \xi<0,
\end{array} \quad y_{p}(\xi, t)=\xi \cos \Theta_{0},\right.
$$

For this piston motion, we obtain the following initial values for shock ray theory,

$$
M_{0}(\xi)=1+\frac{1}{4}(\gamma+1) u_{0} \cos \Theta_{0}, \quad V_{0}(\xi)=-\frac{(\gamma+1) u_{1} \cos \Theta_{0}}{4\left\{1+\frac{1}{2}(\gamma-1) u_{0} \cos \Theta_{0}\right\}}, \quad G_{0}(\xi)=1 .
$$

The initial value for the weakly nonlinear ray theory is

$$
m_{0}(\xi)=1+\frac{1}{2}(\gamma+1) u_{0} \cos \Theta_{0} .
$$




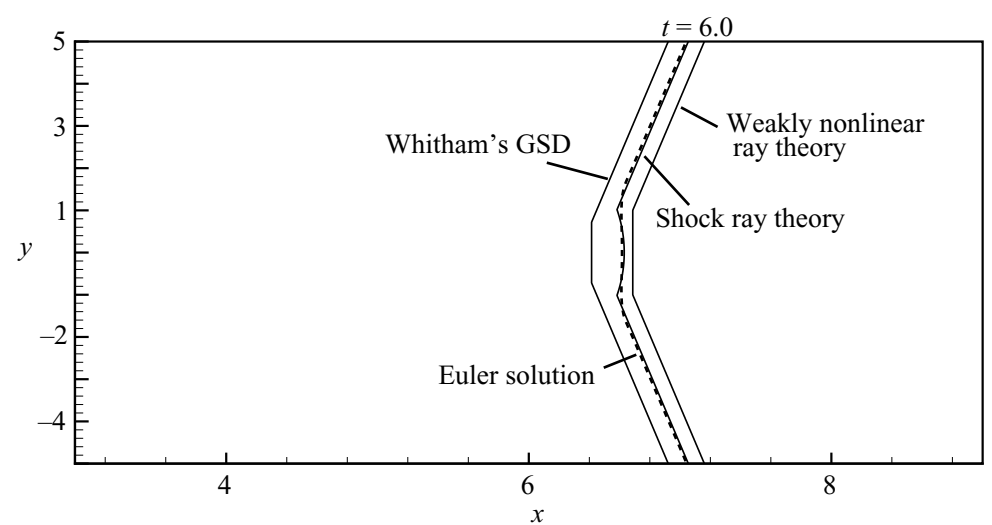

FIGURE 1. Comparison of results for a wedge-shaped accelerating piston with $\Theta_{0}=0.1178$, initial velocity $u_{0}=0.33$ and acceleration $u_{1}=0.15$. $M_{0}=1.2$ and $V_{0}=-0.084375$.

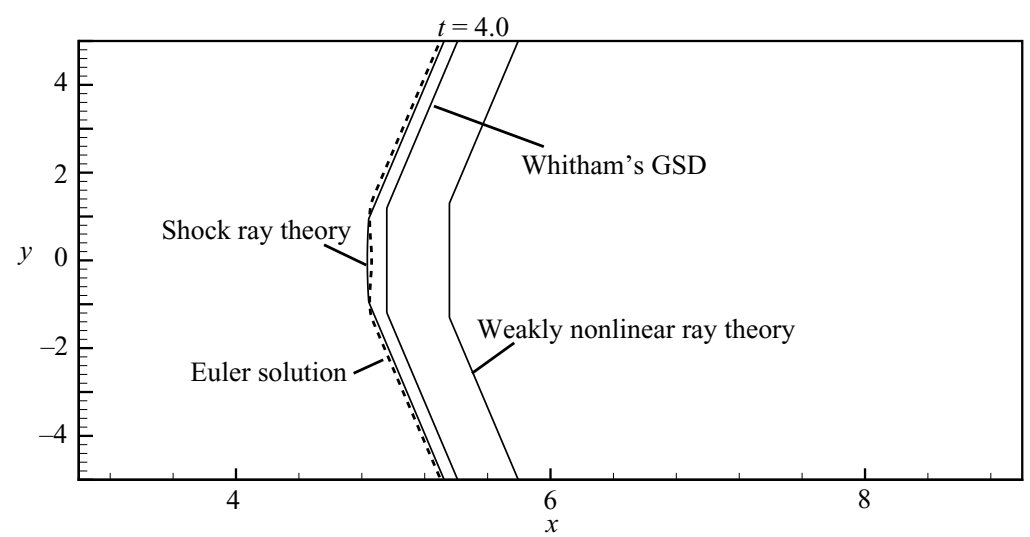

FIGURE 2. Comparison of results for a wedge-shaped decelerating piston with $\Theta_{0}=0.1178$, initial velocity $u_{0}=0.33$ and deceleration $u_{1}=-0.15$. $M_{0}=1.2$ and $V_{0}=0.084375$.

In order that the relation (4.2) is satisfied, we must choose a new $\xi$ in (5.1), which we denote by $\xi_{\text {new }}$ and is given by $\xi_{\text {new }}=\xi /\left(\left(m_{0}-1\right)^{-2} \mathrm{e}^{-2\left(m_{0}-1\right)}\right)$. Let us assume that this has been done for weakly nonlinear ray theory.

5.1. Solution when the wedge-shaped piston is concave to the flow ahead It is easy to find the solution of (4.1)-(4.2) satisfying

$$
m(\xi, 0)=m_{0}(\xi), \quad-\infty<\xi<\infty, \quad \theta(\xi, 0)= \begin{cases}-\Theta_{0}, & \xi>0 \\ \Theta_{0}, & \xi<0\end{cases}
$$

where $0<\Theta_{0}<\pi / 2$ (see expression (6.2.15) in Prasad 2001). Solving (4.3), we obtain the nonlinear wavefront with a pair of kinks joining three straight parts as shown in figures 1 and 2. Note that a kink is an image in the $(x, y)$-plane of a shock in the $(\xi, t)$-plane. We call the central part between the two kinks a 'disk', which is perpendicular to the axis of symmetry, i.e. the $x$-axis. The outer straight parts, we call them 'wings', are parallel to the two sides of the wavefront at $t=0$. 
The initial value $M_{0}$ for GSD is the same as given in (5.2). However, to use the expression (4.4) for $G(m)$, we must use a new $\xi$ in (5.1), as in the case of weakly nonlinear ray theory above. There is an exact solution of this problem also and the graph of the position of the GSD shock front is shown in figures 1 and 2. The general feature of a straight disk joined by two straight wings for a nonlinear wavefront is also present in a GSD shock at $t>0$.

Since an exact solution of the equations of the shock ray theory and Euler's equations are not available, we compute the positions of shock ray theory shock numerically and compare them with positions of shocks by the Euler solution and plot them in the same figures 1 and 2 (see comments on the computation of error in the position of the GSD and shock ray theory shock in the last but one paragraph in $\S 1$ ). We make the following observations from figures 1 and 2 .

(i) The shock fronts by shock ray theory and Euler solution are very close - almost undistinguishable at the times shown.

(ii) The results in figures 1 and 2 correspond to accelerating and decelerating pistons, respectively. Initially, the shocks and nonlinear wavefront start from the same position. However, since the GSD does not take into account the acceleration of the piston, in figure 1 the GSD shock starts falling behind the shock ray theory and the Euler solution shocks which are pushed ahead by the acceleration of the piston. For an accelerating piston with $u_{1}=0.15$, the shock by GSD lags very much behind that by the Euler solution at $t=6$. In the case of a decelerating piston, the GSD shock is ahead of the piston as shown in figure 2, since the deceleration has an effect on the Euler solution and shock ray theory shocks, but not on the shock by GSD.

(iii) The difference between the positions of GSD and shock ray theory will rapidly increase in the case of a decelerating (accelerating) piston because the shock strength of the shock ray theory shock will decrease (increase) owing to the interaction of the shock with nonlinear waves of decreasing (increasing) amplitude coming from the piston at a later time (see the detailed results in Monica \& Prasad 2001).

(iv) The nonlinear wavefront by weakly nonlinear ray theory starts with a larger velocity compared to the shocks by the same piston motion and is always ahead of them. However, the piston acceleration will ultimately push the shock ray theory and Euler solution shocks so much that they will tend to catch up with the nonlinear wavefront, which is self-propagating, i.e. it remains unaffected by the piston acceleration. When the piston is decelerating, the nonlinear wavefront by weakly nonlinear ray theory has moved ahead of the shocks in figure 2 even at $t=4$ as compared to that in figure 1 at $t=6$.

Successive positions of the shock front by shock ray theory have been shown in figure 3. The central disk of shock ray theory and Euler solution shock is convex when observed from the medium ahead of it. This result cannot be observed in GSD shock (or the wavefront by weakly nonlinear ray theory) when the initial shape is in the form of a concave wedge as considered here. If the initial shape were simply concave (but not a wedge) the central disk may become convex owing to local divergence of rays in space not only for Euler solution (Sturtevant 1989) and shock ray theory, but also for weakly nonlinear ray theory (Prasad \& Sangeeta 1999).

The results of this section for the concave piston problem show that shock ray theory is an excellent theory to discuss this type of problem - not only there is a very good agreement with the Euler solution, but it reproduces a very important effect seen in the experiments. 


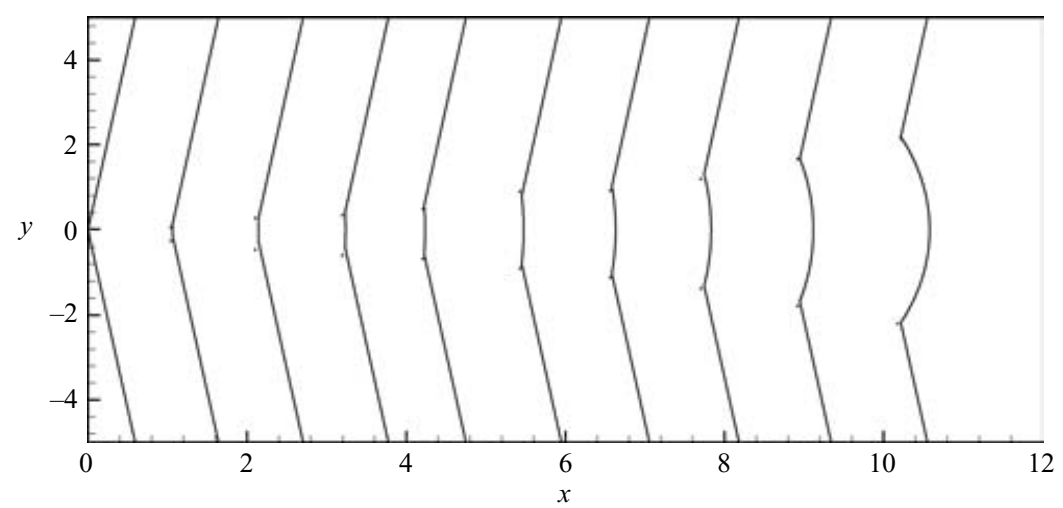

FigURE 3. Successive positions of the shock front using shock ray theory at a time interval 1 $\left(t=0\right.$ to 9 ). The shock is produced by a wedge-shaped piston with $\Theta_{0}=0.1178$, initial velocity $u_{0}=0.33$ and acceleration $u_{1}=0.15 . M_{0}=1.2$ and $V_{0}=-0.084375$.

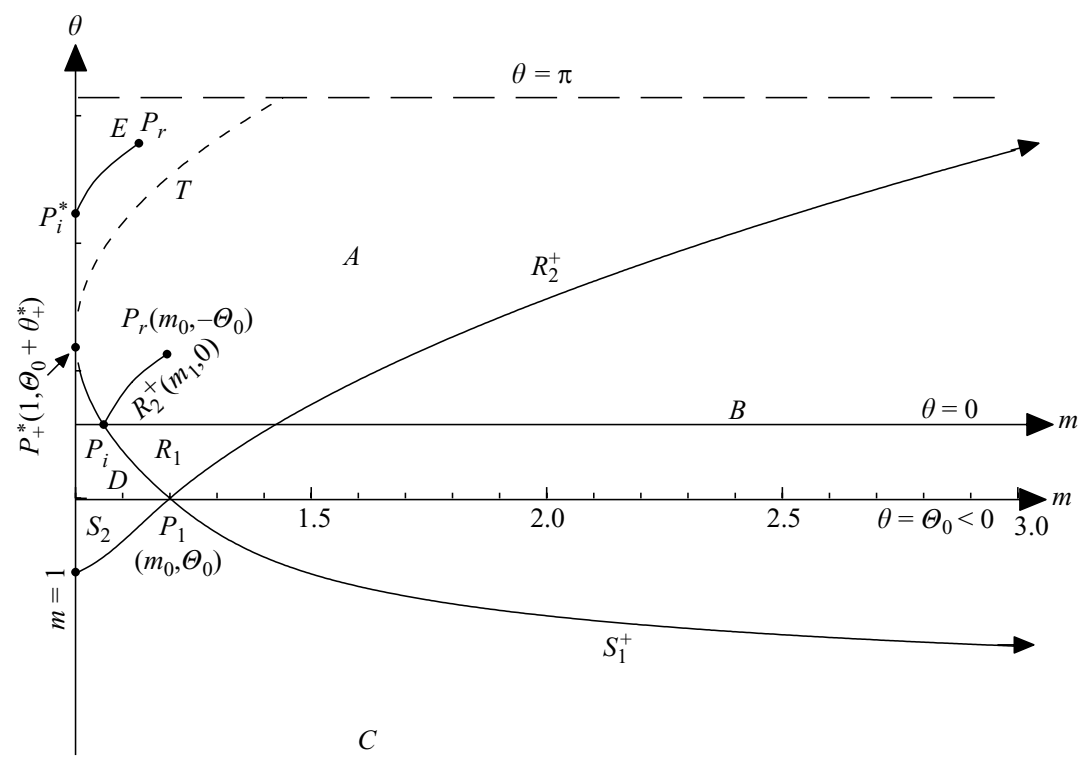

FiguRE 4. Rarefaction and Hugoniot curves in the $(m, \theta)$-plane. Note $\Theta_{0}<0$.

5.2. Solution when the moving wedge-shaped piston is convex to the flow ahead of it

\subsubsection{Solution by WLNRT}

Consider the initial data (5.4) with $-\pi / 2<\Theta_{0}<0$ and $\xi$ normalized suitably as mentioned after (5.3). The piston motion is given by (5.1) with $-\pi / 2<\Theta_{0}<0$. This would correspond to a moving wedge convex to the gas ahead of it.

In order to understand some of the results, we need to reproduce here figure 4 of Baskar \& Prasad (2004), but with slightly changed notation (see figure 4). First, we define rarefaction curves $R_{1}^{-}$and $R_{2}^{+}$as the set of points in the $(m, \theta)$-plane, which can be joined to $\left(m_{0}, \Theta_{0}\right)$ through simple waves of the characteristic families

$$
\frac{\mathrm{d} \xi}{\mathrm{d} t}=\mp \sqrt{\frac{m-1}{2 g^{2}}}
$$


of (4.1)-(4.2). Then,

$$
\begin{aligned}
& R_{1}^{-}\left(m_{0}, \Theta_{0}\right):=\left\{(m, \theta) \mid \theta+\sqrt{8(m-1)}=\Theta_{0}+\sqrt{8\left(m_{0}-1\right)}, 1<m<m_{0}\right\}, \\
& R_{2}^{+}\left(m_{0}, \Theta_{0}\right):=\left\{(m, \theta) \mid \theta-\sqrt{8(m-1)}=\Theta_{0}-\sqrt{8\left(m_{0}-1\right)}, m_{0}<m<\infty\right\} .
\end{aligned}
$$

Similarly, $S_{1}^{+}$and $S_{2}^{-}$are the Hugoniot curves defined with the help of shocks of the first and second family, respectively. $\left(1, \Theta_{0}+\theta_{+}^{*}\right)$ is a point where $R_{1}^{-}$meets the line $m=1$, where

$$
\theta_{+}^{*}=\sqrt{8\left(m_{0}-1\right)}
$$

and $T$ is the $R_{2}^{+}$curve starting from $\left(1, \Theta_{0}+\theta_{+}^{*}\right)$ :

$$
T:\left\{(m, \theta) \mid \theta-\sqrt{8(m-1)}=\Theta_{0}+\theta_{+}^{*}, 1<m<\infty\right\} .
$$

These curves lie on the boundaries of domains $A$ and $E$ in the $(m, \theta)$-plane, as shown in figure 4. For $\left|\Theta_{0}\right|$ sufficiently small, it follows that $\left(m_{0},-\Theta_{0}\right) \in A$ and from the results in Baskar \& Prasad (2004), it follows that the state $P_{r}\left(m_{0},-\Theta_{0}\right)$ on $\xi>0$ (subject to the restriction (5.11) below) can be joined to the state $P_{l}\left(m_{0}, \Theta_{0}\right)$ on $\xi<0$ by the path $P_{l} P_{i} P_{r}$, where $m_{i}$ is such that $P_{r}$ lies on $R_{2}^{+}\left(m_{i}, 0\right)$ (from symmetry it follows that $\theta$ at $P_{i}$ must be zero), where

$$
\sqrt{8\left(m_{i}-1\right)}=\sqrt{8\left(m_{0}-1\right)}-\left(-\Theta_{0}\right)=\sqrt{8\left(m_{0}-1\right)}+\Theta_{0} .
$$

Therefore, the solution of (4.1)-(4.2) with initial data (5.4) (satisfying (5.11) below), $\Theta_{0}<0$, consists of a centred simple wave $R_{1}$ of the first family and another centred simple wave $R_{2}$ of the second family separated by a constant state $\left(m_{i}, \theta=0\right)$. This is the case as long as $-\Theta_{0}$ is not so large as to make the right-hand side of (5.10) negative. Therefore, if $\Theta_{0}$ decreases, it attains a value $\theta_{c}(<0)$ such that the point $P_{r}$, while moving up in figure 4 (actually the $\theta=0$ axis moves up), is on $T$ for $\Theta_{0}=\theta_{c}$ and $P_{i}$ is on the line $m=1$ where $g=\infty$. This means that the weakly nonlinear ray theory is no longer valid. This leads to the conclusion that for a given $m_{0}$, the solution of the weakly nonlinear ray theory for a convex wedge moving in the gas at rest exists if and only if

$$
\Theta_{0}>\theta_{c}\left(m_{0}\right)=-\sqrt{8\left(m_{0}-1\right)} \text { or }\left|\Theta_{0}\right|<-\theta_{c}\left(m_{0}\right) .
$$

Using (5.3), we find $-\Theta_{0}<\sqrt{4(\gamma+1) u_{0} \cos \theta_{0}}$ which finally makes the condition (5.11) for the existence of the solution to be

$$
u_{0}>\frac{\Theta_{0}^{2}}{4(\gamma+1) \cos \Theta_{0}} .
$$

When the solution of the weakly nonlinear ray theory obtained in the $(\xi, t)$-plane is mapped onto the $(x, y)$-plane by (4.3), we find the nonlinear wavefront consists of (see figure 5) two curved parts $E D$ and $B C$ (elementary shapes $\mathscr{R}_{1}$ and $\mathscr{R}_{2}$ as defined by Baskar \& Prasad 2004) separating a straight disk $C D$ (with $m=m_{i}, \theta=0$ ) from two infinite straight wings $B A$ and $E F$. As $\Theta_{0} \rightarrow \theta_{c}+, m_{i} \rightarrow 1$, and the eigenvalues (5.5) of (4.1) tend to zero. The relative displacement in the $(x, y)$-plane of $C$ from $D$ in time $\delta t$ is

$$
g_{i} \delta \xi=g_{i}\left(2 \sqrt{\frac{m_{i}-1}{2 g_{i}^{2}}} \delta t\right)=\sqrt{2\left(m_{i}-1\right)} \delta t,
$$

which tend to zero as $m_{i} \rightarrow 1$. At $t=0$, the distance between $C$ and $D$ is zero, hence it follows that as $\Theta_{0} \rightarrow \theta_{c}+$, the points $C$ and $D$ approach the $x$-axis so that the disk 


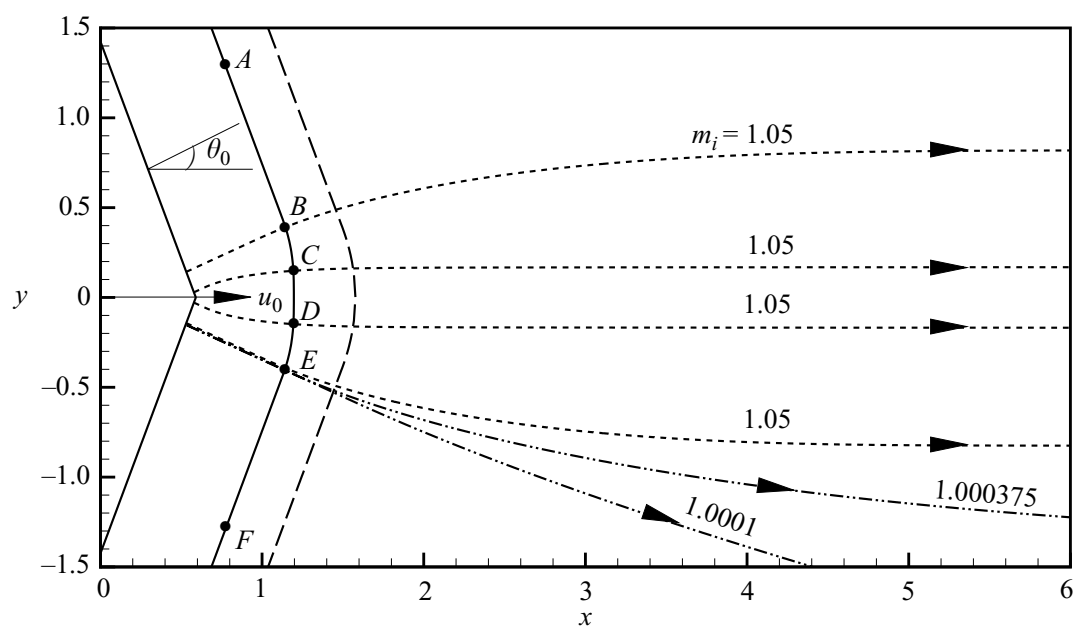

FIGURE 5. The nonlinear wavefront (shown by solid line) $A B C D E F$ at $t=0.6134$ for $m_{0}=1.13$, $m_{i}=1.05$ and $\Theta_{0}=\pi / 8$, consists of two curved parts $B C$ and $E D$ separating a disk $B C$ from the two infinite wings. For the same $\Theta_{0}, m_{0}$ is so chosen that $m_{i}=1.0001$, then the points $C$ and $D$ almost coincide (wavefront shown by long dashes at $t=0.9927$ ) and the rays almost becomes straight as in the case of linear rays.

$C D$ disappears. In this limiting case, the curved part of the nonlinear wavefront near the $x$-axis becomes almost a circle as if drawn by Huygens' method from the corner of the wedge. The central ray along the $x$-axis is a linear ray, but all other rays, though nonlinear, are almost straight, like linear rays, but there is a nonlinear stretching, which is small for rays close to the $x$-axis and large for other rays (depending on the value of $m_{0}$ and their location). We have shown two rays in figure 5 for those cases for which $m_{i}=1.000375$ and $m_{i}=1.0001$.

When $-\Theta_{0}(<\pi / 2)$ is large and satisfies $\Theta_{0}<\theta_{c}\left(m_{0}\right)$, the point $P_{r}\left(m_{0},-\Theta_{0}\right)$ lies above the line $T$ and falls in the domain $E$. The solution of the weakly nonlinear ray theory no longer exists. However, figure 4 still helps us to find the wavefront, which is partly linear and partly nonlinear. From $P_{r}$, we move along the rarefaction curve of the second family up to the point $P_{i}^{*}\left(1, \theta_{i}^{*}\right)$, the rarefaction curve being $R_{2}^{+}\left(1, \theta_{i}^{*}\right)$. From $P_{i}^{*}$, we move along $m=1$ up to the point $P_{+}^{*}$; this corresponds to a linear wavefront. From $P_{+}^{*}$, we move along the rarefaction curve $R_{1}^{-}\left(m_{0}, \Theta_{0}\right)$.

$$
\begin{aligned}
& \text { On } R_{2}^{+}\left(1, \theta_{i}^{*}\right): \theta-\sqrt{8(m-1)}=\theta_{i}^{*}=-\Theta_{0}-\sqrt{8\left(m_{0}-1\right)}, \\
& \text { On } R_{1}^{-}\left(m_{0}, \Theta_{0}\right): \theta+\sqrt{8(m-1)}=\Theta_{0}+\sqrt{8\left(m_{0}-1\right)} .
\end{aligned}
$$

Thus, on the nonlinear part of the wavefront, $\theta$ is a known function of $m$ and we can numerically integrate the ray equations, (4.3), with initial conditions on the piston at $t=0$. This would give the nonlinear part of the wavefront. The linear part of the wavefront, which would be a circle with its centre at the vertex of the wedge, can be obtained by Huygens' method. In the construction of this wavefront, we have avoided using $g$, which tends to infinity as $m \rightarrow 1$.

\subsubsection{Condition for the existence of the solution by shock ray theory}

Consider now the solution of the convex-wedge problem moving along the $x$-axis by shock ray theory. The initial value can be formulated as in (5.1)-(5.2) where we take $-\pi / 2<\Theta_{0}<0$. As indicated at the end of $\S 4$, for a small time, the solution of 
the problem by shock ray theory will be approximately the same as that obtained by a system neglecting the source terms in (2.9)-(2.10). In this case, $(4.8 b)$ for $V$ decouples from (2.8) and (4.8a). These three equations are exactly the same as the equations of the weakly nonlinear ray theory - the only difference is in relating the initial velocity $u_{0}$ to the initial data for $M_{0}(\xi)$ and $m_{0}(\xi)$ as seen in (5.2) and (5.3). Therefore, the critical value $(-\Theta)_{c}=\left|\Theta_{c}\right|$ is given by (following (5.11))

$$
\left|\Theta_{c}\right|=\sqrt{8\left(M_{0}-1\right)}
$$

and the condition $\left|\Theta_{0}\right|<\left|\Theta_{c}\right|$ for the existence of the solution, after using (5.2), gives

$$
u_{0}>\frac{\Theta_{0}^{2}}{2(\gamma+1) \cos \Theta_{0}},
$$

where we note that the right-hand side is positive for $\Theta_{0}<0$.

Once this condition has been satisfied, the solution of the convex-wedge-shaped piston problem by shock ray theory exists. The results obtained from the convex-wedgeshaped piston using shock ray theory will be similar to the results depicted in figures 8 to 10, where we have also plotted the results by the Euler solution and GSD, when the angle between the normals of the two sides of the wedge $=-2 \Theta_{0}=\pi / 2$. Note, an important property from figure 5 is that all rays ultimately become parallel to the axis of symmetry, a result which is purely due to nonlinearity.

\section{Blast wave produced by an explosive placed in a container in the shape of a square}

Let us assume that an explosion of a charge in a container produces a shock front which is initially a square and which has a uniform shock strength. Just behind this shock, we have a family of nonlinear wavefronts which are initially of the same shape and same uniform intensity $w$. For this problem, considering the symmetry in the shape of the piston, it is sufficient to set up an initial-value problem for half of the square (in fact a smaller part of the square will do). In order to see the salient features of the shock front at $t>0$, we first use the weakly nonlinear ray theory to trace the nonlinear wavefront, which was formed at $t=0$ at the piston. In this case, we can obtain an exact solution up to the time ( $t_{c n}$, see figure 6$)$ of interaction of the disturbances from the corners on the same side.

Before we start further discussion, we first give the initial position of the square piston as

$$
\left(x_{p}(\xi, 0), y_{p}(\xi, 0)\right)= \begin{cases}(0, y), & -0.5<y<0, \\ (x, 0), & -0.5<x<0, \\ (-0.5, y), & -0.5<y<0, \\ (x,-0.5), & -0.5<x<0 .\end{cases}
$$

The length of a side of the piston is 0.5 .

We assume each side of the piston suddenly starts moving with a speed $u_{0}$ and acceleration $u_{1}>0$.

\subsection{Weakly nonlinear ray theory solution}

The initial Mach number of the piston is given by $m_{0}=1+(\gamma+1) / 2 u_{0}$. The upper half of the piston, which we consider for setting up the initial-value problem is a portion of the initial piston from $P_{1}$ to $P_{5}$, as shown in the inner square of figure 7 . 


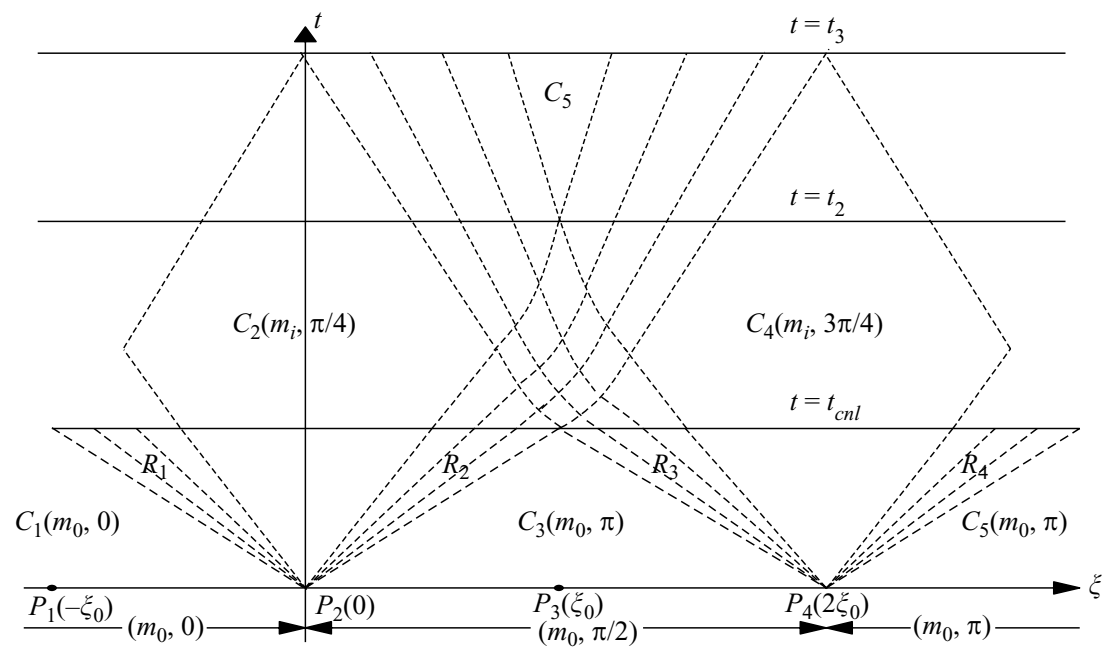

FIGURE 6 . When $m>m_{c}$ and $t<t_{c n l}$, the solution by weakly nonlinear ray theory consists of a number of centred simple waves $R_{1}, R_{2}, R_{3}, \ldots$ separated by constant state regions $C_{1}, C_{3}, C_{5}, \ldots$

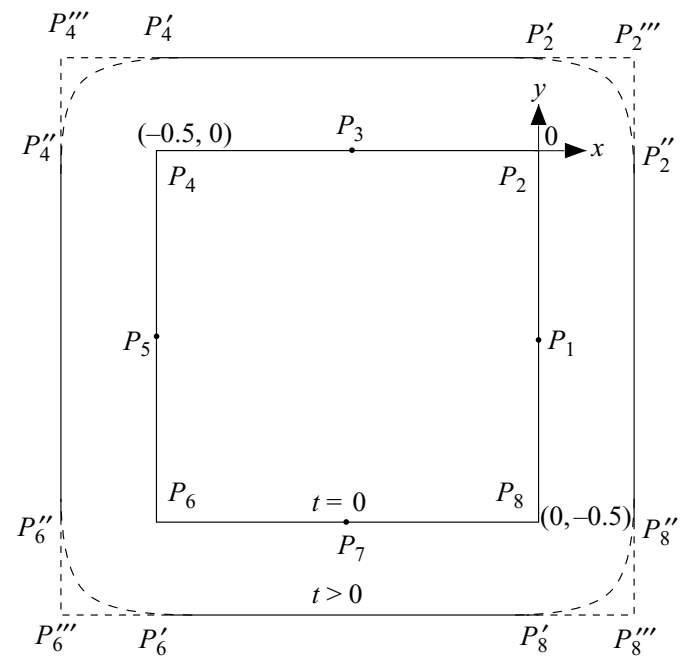

FIGURE 7. Piston motion. (a) Sides of the square have fixed length and they simply move leaving four gaps $P_{2}^{\prime \prime} P_{2}^{\prime}, P_{4}^{\prime \prime} P_{4}^{\prime}$, etc. $(b)$ The lengths of all sides increase giving a bigger square $P_{2}^{\prime \prime \prime} P_{4}^{\prime \prime \prime} P_{6}^{\prime \prime \prime} P_{8}^{\prime \prime \prime}$.

This results in the following initial value for the system (4.1)-(4.2)

$$
m(\xi, 0)=m_{0}, \quad-\xi_{0}<\xi<3 \xi_{0}, \quad \theta(\xi, 0)= \begin{cases}0, & -\xi_{0}<\xi<0 \\ \pi / 2, & 0<\xi<2 \xi_{0} \\ \pi, & 2 \xi_{0}<\xi<3 \xi_{0}\end{cases}
$$

where $\xi_{0}$ is chosen in such a way that when $\xi$ varies in $\left(-\xi_{0}, 0\right)$, the point $\left(x_{p}(\xi, 0)\right.$, $\left.y_{p}(\xi, 0)\right)$ moves on the line $x=0$ from $P_{1}$ to $P_{2}$; when $\xi$ varies in $\left(0,2 \xi_{0}\right)$ the point moves on the line $y=0$ from $P_{2}$ to $P_{4}$; and when $\xi$ varies in $\left(2 \xi_{0}, 3 \xi_{0}\right)$, the point moves 
on the line $x=-0.5$ from $P_{4}$ to $P_{5}$. Then

$$
\xi_{0}=\frac{1}{4\left(m_{0}-1\right)^{-2} \mathrm{e}^{-2\left(m_{0}-1\right)}}=\frac{1}{4 g_{0}} .
$$

If $s$ is the arclength along the initial boundary measured from the point $P_{2}$, then $\xi=4 \xi_{0} s$.

The condition (5.11) for the existence of the solution in terms of a critical angle $\theta_{c}$ can also be written in terms of a critical Mach number $m_{c}$. Comparing the geometry of the wedge given by (5.1), we find the jump in the direction of the normal to be $2 \Theta_{0}=\pi / 2$. Hence, the critical Mach number is

$$
m_{c}=1+\frac{\pi^{2}}{128}
$$

and for $m_{0}>m_{c}$, we have $m_{i}>1$. Thus, a necessary and sufficient condition for the existence of the solution by the weakly nonlinear ray theory is $m>m_{c}$. Considering the solution for small time by shock ray theory, the corresponding condition, (5.15), can be written in terms of a critical Mach number $M_{c}$ of the shock $M_{c}=1+\pi^{2} / 128$ and the solution of the shock ray theory equations exists only if $M_{0}>M_{c}$. This critical Mach number can easily be translated into a critical speed of the piston (see (5.16)).

When $m>m_{c}$, we can find an exact solution of the problem by weakly nonlinear ray theory for $t<t_{c n l}$, where $t_{c n l}$ is the time when the waves moving on the nonlinear wavefront from the two corners $P_{2}$ and $P_{4}$ meet. This, in fact, is the time when, starting from $P_{2}$, the leading end of the central simple wave of the positive characteristic family meets the line $\xi=\xi_{0}$ in the $(\xi, t)$-plane (see figure 6). For $t<t_{c n l}$, the solution in the $(\xi, t)$-plane consists of isolated rarefaction waves $R_{1}, R_{2}, R_{3}, \ldots$ (of the same strength) separated by constant states with the same value of $(m, \theta)=\left(m_{i}, \theta_{i}\right)$, with $m_{i}<m_{0}$ and from symmetry, it follows that $\theta_{i}=\pi / 4,3 \pi / 4, \ldots$ It is easy to determine an equation which would determine $m_{i}$.

For $t>t_{c n l}$, no exact solution of the problem can be found and we must solve the problem numerically. Starting from $t=t_{c n l}$, the two rarefaction waves, say, $R_{2}$ and $R_{3}$ (of different families, as shown in figure 6) start interacting. From the general theory in Baskar \& Prasad (2004), it follows that these interactions will be of finite duration from time $t_{c n l}$ to $t_{2}$, leading again to two rarefaction waves of two different families from each interaction. Meanwhile, the newly generated rarefaction waves from interactions will bound the constant-state regions between $R_{1}$ and $R_{2}$, etc. up to a time $t_{3}$. The solution beyond $t_{3}$ will again consist of non-constant regions and constant-state regions between the two rarefaction waves produced as a result of interaction of $R_{2}$ and $R_{3}$, etc. such as $C_{5}$.

Using the characteristic velocity (5.5) and (6.3), we find the value of $t_{c n l}$ from

$$
t_{c n l}=\frac{\xi_{0}}{\sqrt{(m-1) / 2 g^{2}}}=\frac{1}{\sqrt{8\left(m_{0}-1\right)}} .
$$

For the initial velocity $u_{1}$ of the piston to be 0.333 , as taken in figures 8 to 10 , we find $m_{0}=1.4$ and therefore from (6.5), we have $t_{c n l}=0.55902$. The shape of a weakly nonlinear wavefront, as it propagates, is similar to that of the shock front shown in figure 14, it was also observed to be nearly circular for $t \geqslant t_{c n l}$.

\subsection{GSD solutions}

The main difference in weakly nonlinear ray theory and GSD theory is that in the expressions (4.2), the metric $g$ and Mach number $m$ are replaced by the expressions 

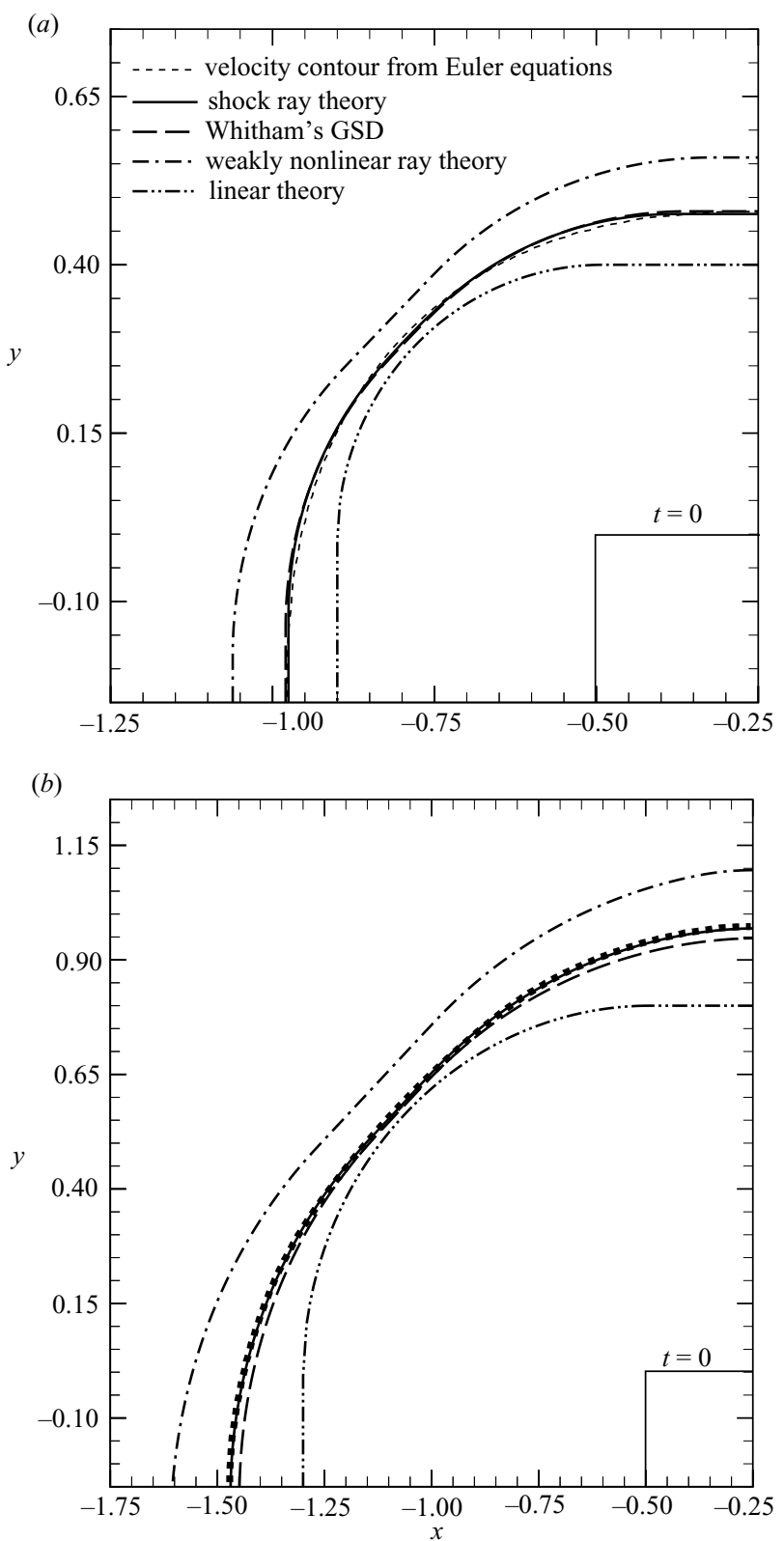

FIgURE 8. Comparison of results at time $(a) t=0.4$ and $(b) t=0.8$ in the case of a blast wave with an accelerating piston with initial velocity $u_{0}=0.333$ and acceleration $u_{1}=0.5$.

(4.4) and (2.2) for $G$ and $M$, respectively, leading to corresponding changes in expressions such as (6.5). All qualitative features of the solution of weakly nonlinear ray theory are also seen in the solution of GSD.

6.3. Interpretation of the initial conditions for the weakly nonlinear ray theory, GSD and shock ray theory

The above features of the solutions by weakly nonlinear ray theory and GSD will also be present in the solution by shock ray theory in a modified form. However, are 


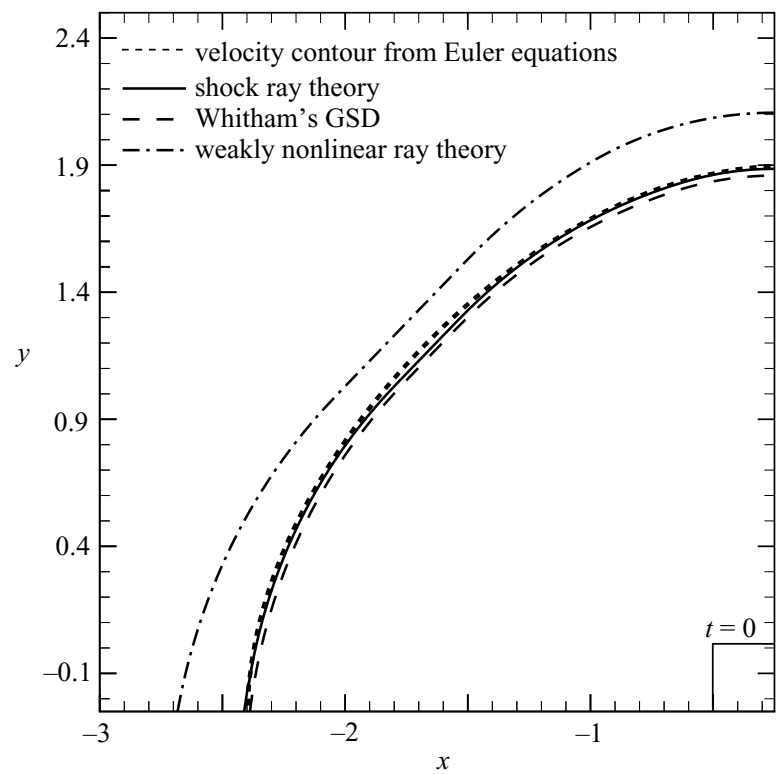

FIGURE 9. A long time comparison of results at time $t=1.6$ in the case of an accelerating piston with initial velocity $u_{0}=0.333$ and acceleration $u_{1}=0.5$.

these common features from all three theories shared by the solution of the original problem, i.e. by the Euler solution? This question becomes important because there appears to be more than one initial data set for Euler's equations which lead to the same initial-value problem for any one of the three theories: weakly nonlinear ray theory, GSD and shock ray theory.

Consider the following two blast-wave problems produced by a piston initially in the form of a square. The shapes of the piston for $t>0$ are shown in figure 7. (a) In the first problem, the lengths of the sides remain fixed as they move with same speed and, at a later time $t>0$, we obtain a punctured square with gaps $P_{2}^{\prime \prime} P_{2}^{\prime}, P_{4}^{\prime} P_{4}^{\prime \prime}, P_{6}^{\prime \prime} P_{6}^{\prime}, P_{8}^{\prime} P_{8}^{\prime \prime}$ at the corners. $(b)$ The lengths of all sides increase as they move so that, at a time $t>0$, we obtain a bigger square $P_{2}^{\prime \prime \prime} P_{4}^{\prime \prime \prime} P_{6}^{\prime \prime \prime} P_{8}^{\prime \prime \prime}$. We can have one more problem in which the corners of the expanding square are rounded, as shown in the figure 7 . All these problems lead to the same initial data for weakly nonlinear ray theory, or GSD or shock ray theory, but for the Euler solution we must prescribe different boundary conditions. In problem $(a)$, a vacuum is created in the gap and suitable boundary conditions are to be provided for Euler's equations. Similarly, in the case of (b), the fluid at the corners is continuously pushed and a different type of boundary condition is required. The two problems $(a)$ and $(b)$ have different Euler solutions, but the corresponding problem either for weakly nonlinear ray theory or GSD or shock ray theory has same solution.

The weakly nonlinear ray theory shows that from the corner $P_{2}$, we have two elementary shapes (images of elementary waves) corresponding to $R_{1}$ and $R_{2}$ elementary waves. They are separated by a straight part carrying the value $m_{i}$ (i.e. a perturbation amplitude $w_{i}$ given by $w_{i}=2\left(m_{i}-1\right) /(\gamma+1)$, see the relation (4.2)) and have normal direction given by $\theta_{i}=\pi / 4$. What should be the correct boundary condition at the piston for Euler's equations corresponding to this solution of weakly nonlinear ray theory? We note that weakly nonlinear ray theory is valid for small $m-1$, but there is a critical value $m_{c}$ (slightly greater than 1 , as seen from (6.4)) which is the lowest 

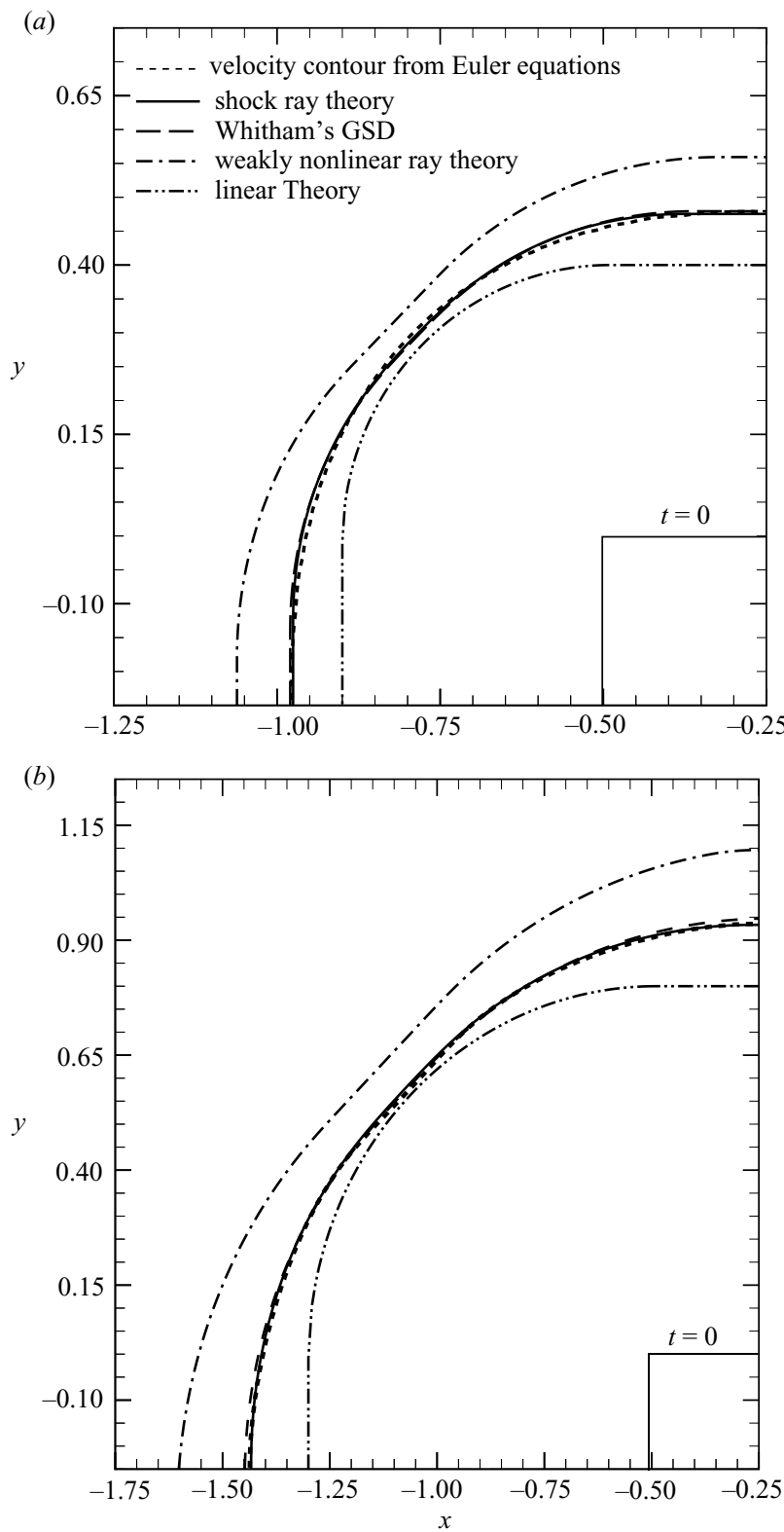

FIGURE 10. Comparison of results at time $(a) t=0.4$ and $(b) t=0.8$ in the case of a blast wave with a decelerating piston with initial velocity $u_{0}=0.333$ and deceleration $u_{1}=-0.5$.

value of $m_{0}$ and for this $m_{i}=1$ or $w_{i}=0$. For some admissible values of $m_{0}$ (see table 1) and for $\Theta_{0}=\pi / 4$, we find that the values of $m_{i}-1$ are quite small. Thus, the values of $m_{i}$ obtained in weakly nonlinear ray theory correspond to still smaller piston speed at the corner. Moreover, for a small piston speed, the boundary conditions for the Euler solution are applied to the piston fixed at its initial position. Hence, we think that the most appropriate boundary value at $P_{2}$ (and hence at other corners) is zero velocity of the fluid (and hence the piston). Since the domain in the $(\xi, t)$-plane just opposite to $P_{2}$ is $C_{2}$ (see figure 6), where the solution is constant, the most 


\begin{tabular}{ccccc}
\hline$m_{0}$ & 1.1 & 1.15 & 1.2 & 1.25 \\
$m_{i}$ & 1.001486 & 1.012016 & 1.028742 & 1.049426 \\
& & TABLE $1 . \Theta_{0}=\pi / 4$. \\
\hline
\end{tabular}

appropriate value of the acceleration at $P_{2}$ should also be zero. At all other points, we can prescribe the initial fluid speed $1+w$ which is the same as the initial piston speed $\left|\left(x_{p t}(\xi, 0), y_{p t}(\xi, 0)\right)\right|$.

From the general theory on the Riemann problem for kinematical conservation laws (Baskar \& Prasad 2004), it follows that $m_{i}$ in region $C_{2}$ in figure 6 satisfies $m_{i}<m_{0}$. Similarly, from the result on interaction of two centred waves in the same paper, the value $m_{i i}$ in $C_{5}$ satisfies $m_{i i}<m_{i}$. Hence, after the two centred waves from the corners of the square have completed interaction, the Mach number $m_{i i}$ directly above the side $P_{2} P_{4}$ is smaller than anywhere else. Hence, the nonlinear wavefront opposite to the corners now moves faster than that directly opposite to the sides of the square. This clearly explains the reason for the evolution towards a circular shock (discussed at the end of this section).

\subsection{Comparison of the Euler solution and solutions by GSD and shock ray theory}

As discussed above, we apply the appropriate boundary conditions for the Euler solution on the initial position of the square piston and solve Euler's equations. Given the piston motion, we can set up the initial values for the equations of shock ray theory from (3.5) and (3.12). For shock ray theory, we take $\xi$ to be the arclength from $P_{2}$ and hence $G_{0}=G(\xi, 0)=1$.

Before we discuss a comparison of the results, we calculate $t_{c s}$, the time when the waves from the corners $P_{2}$ and $P_{4}$ (figure 7 ) meet at a point above $P_{3}$ according to shock ray theory. The relevant eigenvalue (or characteristic velocity) of the system (2.5)-(2.6) is $\sqrt{(M-1) /\left(2 G^{2}\right)}$ in the $(\xi, t)$-plane. Since the wave from $P_{2}$ moves into the constant state with $M=M_{0}$ and $G_{0}=1$, it reaches $\xi=1 / 4$, i.e. a point above the point $P_{3}$ in time

$$
t_{c s}=\frac{1}{\sqrt{8\left(M_{0}-1\right)}} .
$$

Since $0<M_{0}-1<m_{0}-1$, it follows from (6.5) and (6.6) that

$$
t_{c n l}<t_{c s} \text {. }
$$

For the value $M_{0}=1.2$, we find $t_{c s}=0.79057$. This gives an order of time when we may consider the shock front to be approximately circular. We shall comment on this later in this section.

Figures 8 to 10 contain graphical depiction of the results by all five theories mentioned in this paper. Though we have drawn the graphs of results by the weakly nonlinear ray theory and linear theory also, the important comparison is between the results by shock ray theory, GSD and Euler solution. Figures 8 to 9 contain results for an accelerating piston at time $t=0.4, t=0.8$ and $t=1.6$, respectively. All three shocks start with the same initial position and the same initial velocity and hence for small $t$, they almost overlap. At time $t=0.4$, figure $8(a)$ shows that all three curves representing the results by the three theories almost overlap, but the shock ray theory curve occupies overall a middle position of the GSD and Euler solution curve. At $t=0.8$ (figure $8 b$ ), the shock ray theory and Euler solution overlap as at $t=0.4$, but the GSD curve now lags behind and this effect becomes more pronounced at $t=1.6$ 


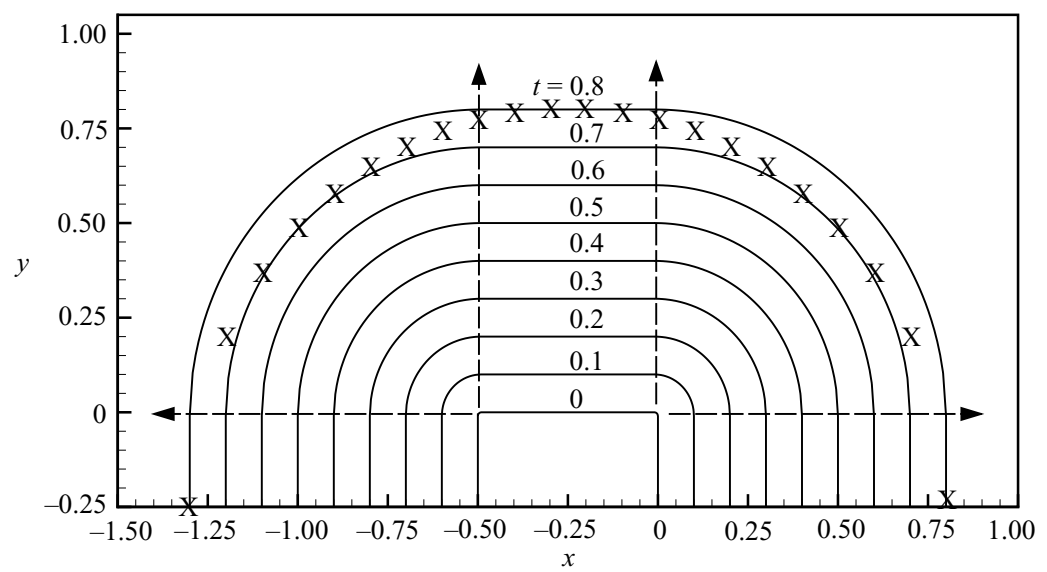

FIGURE 11. Successive positions at times $t$ of a leading wavefront from a blast wave due to a square shaped source using linear theory. A circle (shown by the symbol X) of an appropriate radius and centre at the centre of the square has been compared with the linear wavefront at $t=0.8$.

(figure 9). For an accelerating piston, energy is fed into the flow at an increasing rate, but this increasing input of energy is not taken into account by GSD. This causes the GSD shock to lag behind. We stopped the comparison at $t=1.6$ because beyond this time, as $t$ increases, the GSD result is bound to differ significantly from that of shock ray theory. Figures $10(a)$ and $10(b)$ contain results of a decelerating piston at $t=0.4$ and $t=0.8$, respectively. At both times, all three results are very close, but the shock ray theory curve lies almost in the middle of the other two curves. In the decelerating piston case, the energy is fed into the flow at a decreasing rate. Up to the time we have presented our results, the deceleration has not sufficiently affected the relative positions of shocks and this has caused the GSD shock to be only a little ahead of the shock ray theory and the Euler solution shocks. It is well known that GSD does give good result in some cases (see Whitham 1974), but it is only accidental (Prasad, Ravindran \& Sau 1991). What is important for us to note is that shock ray theory gives consistently good results, very close to Euler's solutions, not only in this case, but also for the wedge-shaped-piston problem discussed in the previous section (figures 1 and 2). This agrees with the conclusion of Kevlahan (1996), who compared the results of the shock ray theory with the Euler solution and found excellent agreement between the two results. He also found excellent agreement of the results of shock ray theory with the experimental results of Sturtevant \& Kulkarni (1976) and some known exact solutions.

\subsection{Evolution toward a circular shock}

We now discuss the second aim of this paper. The shock front (more precisely the linear wavefront) produced by a square piston, when calculated according to the linear theory will tend to a circle as $t \rightarrow \infty$. At any finite time, it will have four straight parts (obtained easily by using the linear ray theory) joined by circular arcs (arising from corners and obtained by Huygens' method) as shown in figure 11. If $a$ is the length of the side of the square at $t=0$, the ratio of the total length of the straight parts of the linear wavefront to that of the circular arcs is $2 a /(\pi t)$. Here, $a=0.5$ and, therefore, at $t=0.8$, this ratio in figure 11 is approximately $1 / 3$. The linear wavefront may be treated as almost circular, when this ratio is $1 / 10$, i.e. $t=O(20 a / \pi)$ which 


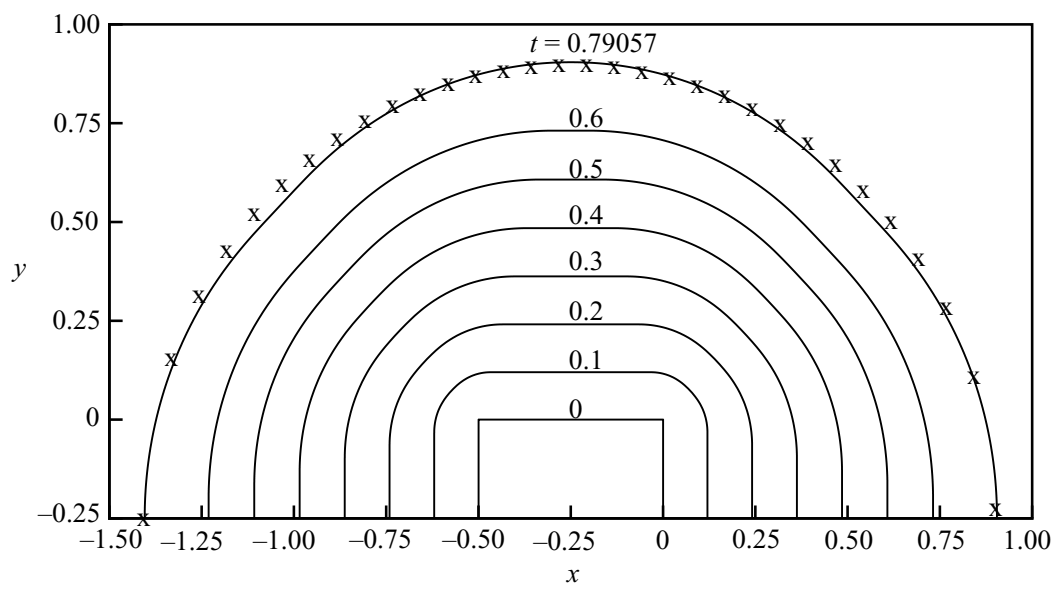

FiguRE 12. Successive positions of a leading shock front (at different times $t$ ) from a blast wave due to a square shaped source using shock ray theory with $u_{0}=0.333, u_{1}=0.5$. A circle of an appropriate radius and centre at the centre of the square has been shown to compare with the shock front at $t=t_{c}$ by the symbol X. $M_{0}=1.2$ and $V_{0}=-0.28125$.

is equal to $\approx 3$. Figure 12 shows the successive positions of the shock ray theory shock up to a time 0.79057 which is quite close to $t_{c}$. At this time, a circle (shown by the symbol ' $\mathrm{X}$ ') has been drawn with its centre at the centre of the square. The shock and the circle are almost coincident. Whereas, figure 11 shows that the linear wavefront deviates very much from an appropriate circle. This evolution almost into a circle of an initially square shock has taken place at $t_{c}$ which is just a third of the time when the linear wavefront may be treated as a circle. The reasons for the shock to tend to become a circle are the nonlinear waves on the shock, which move with the characteristic velocities (or eigenvalues) of the hyperbolic system (2.5)-(2.6). This tendency for a convex shock front to become smooth is nothing but corrugation stability of a plane shock front (Monica \& Prasad 2001) and is explained at the end of the $\S 7$. The interaction of nonlinear waves on the shock front, as noted while discussing the results of weakly nonlinear ray theory in figure 6, plays an important role.

Some characteristic properties of the shock produced by a square piston have been shown graphically in figures 13 and 14. Figure 13 does not give good resolution of very small constant-state regions at the original four corners. This has been shown by a graph of $\Theta$ with $\xi$ in a small neighbourhood of $\xi=0$ in figure 14. This corresponds to the constant-state region $C_{2}\left(m_{i}, \pi / 4\right)$ in figure 6 . Figure $13(d)$ shows that the value of $G$ is very large in a small neighbourhood of $\xi=0$, which implies that a very small neighbourhood of $\xi=0$ where $\Theta$ is constant is mapped onto a straight part of the shock as seen in figure 12, where the shock front deviates a little from the circle.

\section{Shock produced by a wavy piston}

Results on the propagation of a shock initially in a periodic shape have been discussed by Monica \& Prasad (2001). In this paper, we present numerical results for only two cases, but first, we give an extension of the result (6.4). In fact, this extension is rewriting the result (5.15) in terms of the critical Mach number $m_{c}$, as explained for (6.4). Consider the initial position of a nonlinear wavefront, which consists of two 
(a)

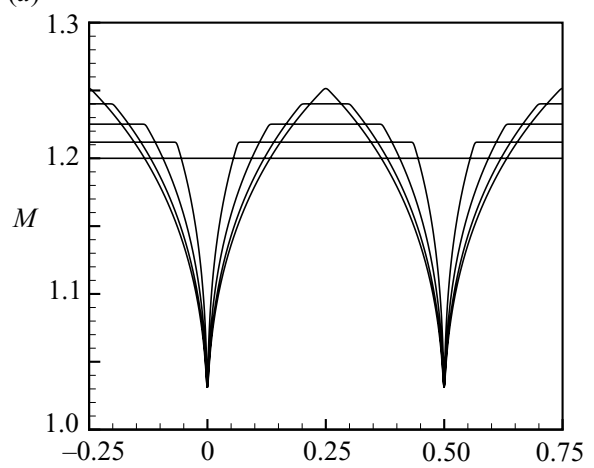

(c)

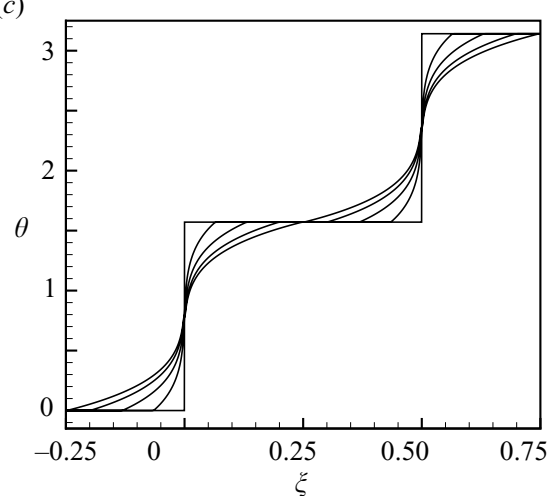

(b)
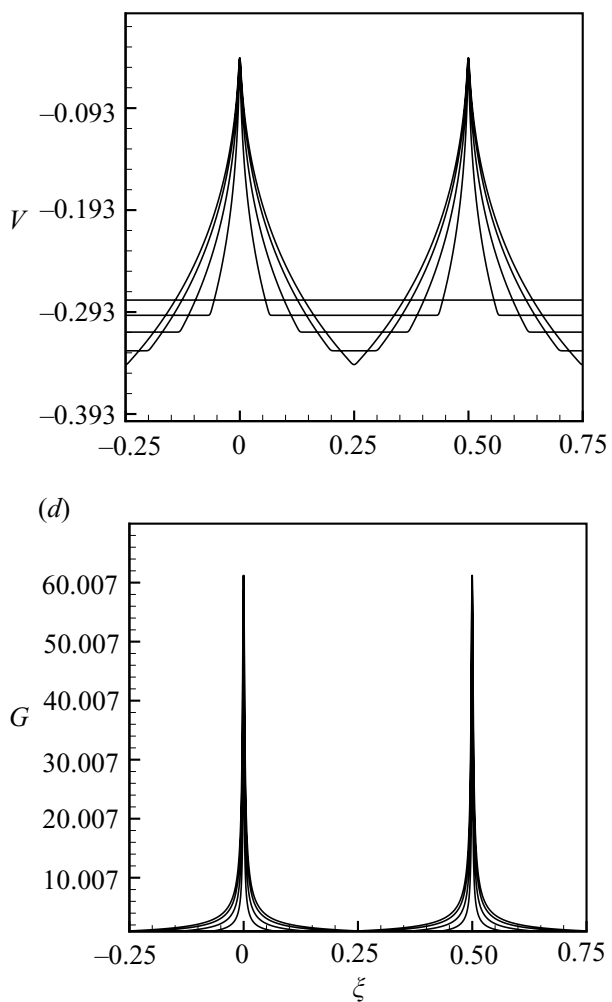

Figure 13. Variation in $M, N, G$ and $\theta$ with respect to $\xi$ in the case of a blast wave from a square shaped source using shock ray theory. The innermost curves correspond to the results at $t=0$ and the outermost ones to those at $t=0.79057$. Here, $u_{0}=0.333$ and $u_{1}=0.5$.

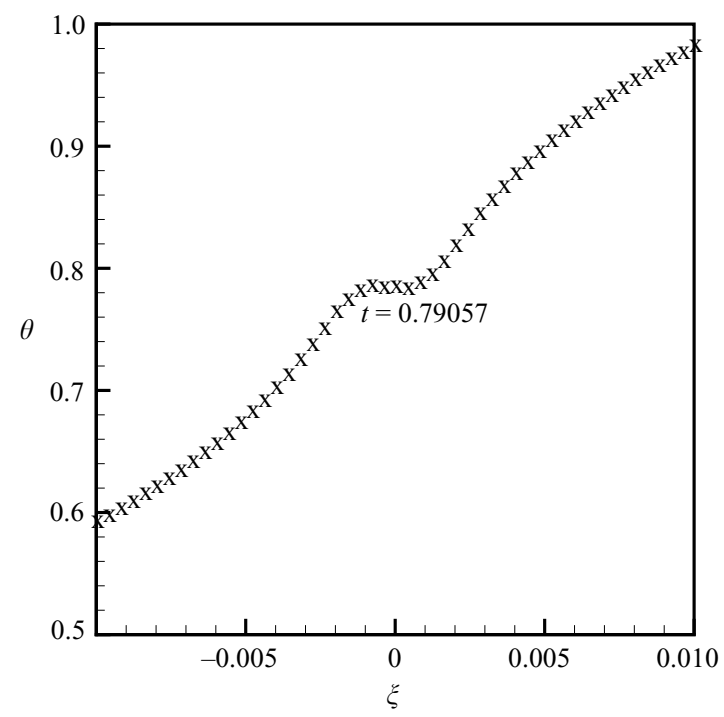

FIGURE 14. An enlarged $\theta$ graph presented in figure 13(c). 
infinite straight parts meeting at a point. Let $(m, \theta)$ on the lower part be $\left(m_{0}, 0\right)$ and that on the upper part be $\left(m_{0}, \theta_{r}\right)$, with $0<\theta_{r}<\pi$. The extension of the result (6.4) is the existence of a critical number $m_{c}$ :

$$
m_{c}\left(\theta_{r}\right)=1+\frac{1}{32} \theta_{r}^{2},
$$

such that if $m_{0}>m_{c}$, the solution of the Riemann problem for (4.1)-(4.2) with initial condition $(m, \theta)=\left(m_{0}, 0\right)$ for $\xi<0$ and $=\left(m_{0}, \theta_{r}\right)$ for $\xi>0$ exists and is unique. $m_{c}$ increases monotonically from 1 to $1+\pi^{2} / 32=1.3084$ as $\theta$ varies from 0 to $\pi$.

Consider now a piston whose shape is in the form of a periodic curve which is formed by periodically extending in the $y$-direction a finite wedge given by

$$
x=\left\{\begin{array}{l}
x_{0}+y \tan \Theta_{0}, \quad-x_{0} \cot \Theta_{0}<y<0, \\
x_{0}-y \tan \Theta_{0}, \quad 0<y<x_{0} \cot \Theta_{0},
\end{array}\right.
$$

where $\Theta_{0}$, a constant satisfying $0<\Theta_{0}<\pi / 2$, is the angle which the normal to the upper part of the wedge makes with the $x$-axis. We choose $\xi$ to be the arclength along the piston measured from the corner $\left(x_{0}, 0\right)$ of the piston. Then, the corners of the piston above $\left(x_{0}, 0\right)$ in one period are at $\left(0, x_{0} \cot \Theta_{0}\right)$ and $\left(x_{0}, 2 x_{0} \cot \Theta_{0}\right)$ and correspond to $\xi=x_{0}\left(1+\cot ^{2} \Theta_{0}\right)^{1 / 2}=\xi_{1}$, say, and $\xi=2 \xi_{1}=\xi_{2}$, say. Similarly, the corners of the piston below $\left(x_{0}, 0\right)$ in the lower period are at $\left(0,-x_{0} \cot \Theta_{0}\right)$ and $\left(x_{0},-2 x_{0} \cot \Theta_{0}\right)$ and correspond to $-\xi_{1}$ and $\xi_{2}$.

The shock front, produced by the piston, will initially coincide with the piston so that the change in the angle of the normal at the corner $\left(x_{0}, 0\right)$ is $2 \Theta_{0}$. Therefore, the value of $M_{0}$, the constant Mach number of the shock given by (3.5) should (following (7.1)) satisfy $M_{0}>1+\Theta_{0}^{2} / 8$ for the existence of the solution. by

The angle $\Theta$ between the normal to the shock front and the $x$-axis is initially given

$$
\Theta(\xi, 0)= \begin{cases}\Theta_{0}, & \text { if }-\xi_{2}<\xi \leqslant-\xi_{1}, \\ -\Theta_{0}, & \text { if }-\xi_{1}<\xi \leqslant 0, \\ \Theta_{0}, & \text { if } 0<\xi \leqslant \xi_{1}, \\ -\Theta_{0}, & \text { if } \xi_{1}<\xi \leqslant \xi_{2},\end{cases}
$$

and periodically extended for $\xi<-\xi_{2}$ and $\xi>\xi_{2}$. The above initial condition corresponds to three Riemann problems in the intervals: 1. $\left(-\xi_{2},-\xi_{1}\right)$ and $\left(-\xi_{1}, 0\right)$; 2. $\left(-\xi_{1}, 0\right)$ and $\left(0, \xi_{1}\right) ; 3 .\left(0, \xi_{1}\right)$ and $\left(\xi_{1}, \xi_{2}\right)$. The solution of the non-homogeneous system (2.8)-(2.10) for a small time approximates the solution of the corresponding system of four conservation laws (2.8) and (4.8). To the reduced system, the analysis of Baskar \& Prasad (2004) of the Riemann problem for the kinematical conservation laws (presented in the first part of $\S 5.2$ in this paper) would apply. Later on, the centred waves, which emerge out of the reduced equations as shown in figure 6 , would be modified by the source terms, and waves from other periods would come and interact. This would result in corrugational stability leading to the formation of a smooth shock front at a large time (Monica \& Prasad 2001). From the corners in the centre of the convex parts (such as that at $\xi=0$ ) two centred rarefaction waves would emerge. Similarly, from the corners in the concave parts (such as those at $-\xi_{1}$ and $\xi_{1}$ ), two shocks emerge. Later on, they will go through multiple interactions leading to a series of complex shapes of the shock front as the time $t$ increases (see figure 15).

In figure 16, we have shown the comparison between the GSD, shock ray theory and the weakly nonlinear ray theory. As observed in the previous cases for an accelerating piston, the shock front from GSD remains behind the shock ray theory shock and the wavefront from weakly nonlinear ray theory travels ahead of it. From the comparison 

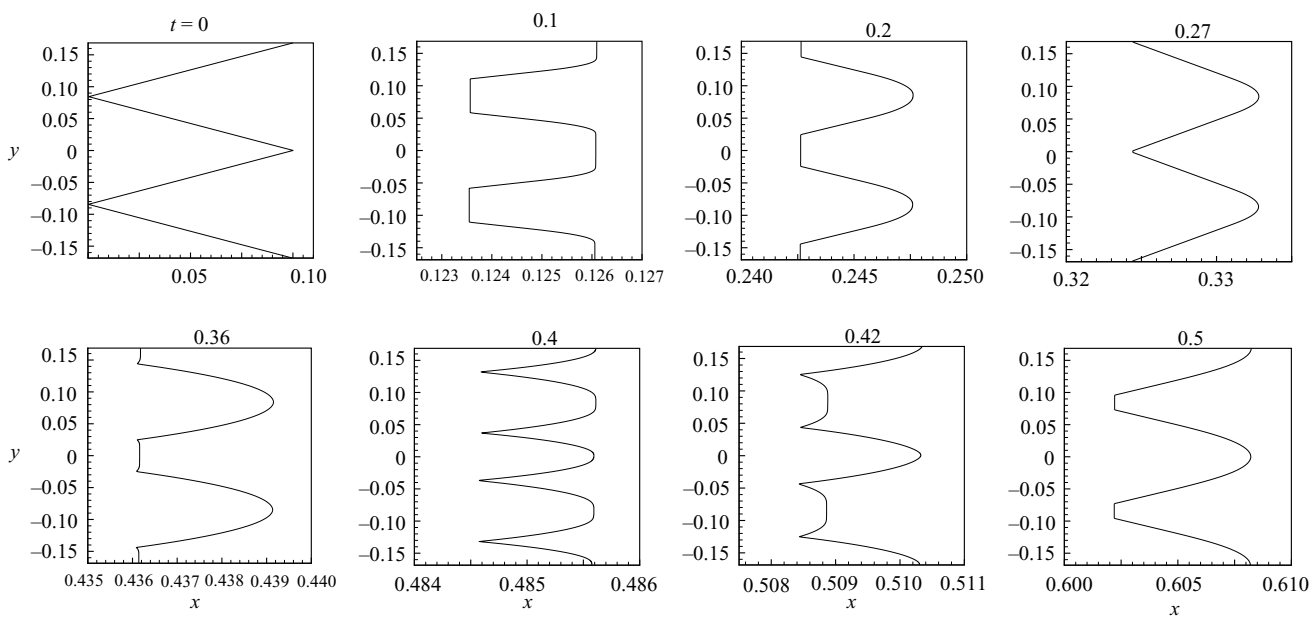

FIGURE 15. Successive positions of a shock, produced by an accelerating piston of periodic shape, using shock ray theory with $u_{0}=0.333$ and $u_{1}=0.5$.

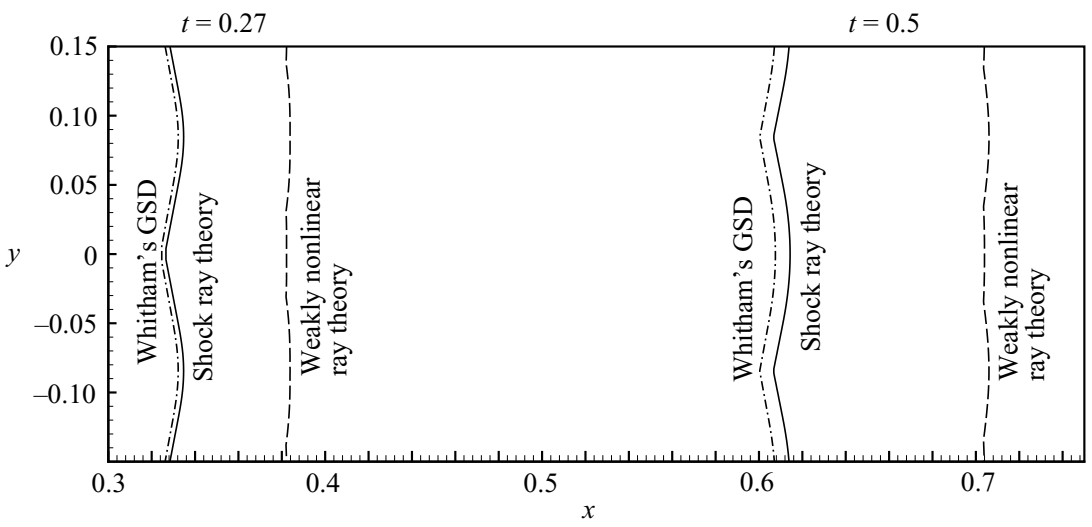

FIgURE 16. Comparison of results in the case of a periodic shock front with $u_{0}=0.333$ and $u_{1}=0.5$.

of results with Euler equations of the previous sections, we argue that the Euler solution and shock ray theory would be close. Therefore, although qualitatively the shape of the shock front from GSD is the same, the shock position remains behind the shock from shock ray theory and therefore from full gas dynamics equations. It is also observed (in the numerical solution not presented here) that when we increase the acceleration of the piston, the shock front from shock ray theory comes closer to the wavefront obtained from weakly nonlinear ray theory and hence travels far ahead of GSD. Finally, after a long time, the effect of acceleration of the piston will dominate and the shock ray theory and Euler solution shocks would move far ahead of the GSD shock and the weakly nonlinear ray theory wavefront.

\section{Conclusion}

We have derived a new set of conservation forms of the first two compatibility conditions of shock ray theory for a weak shock. These conservation forms are more natural and follow a pattern which can be easily extended for each one of the 
infinite set of compatibility conditions for a weak shock. Hence, it is possible to write conservation forms of higher-order shock ray theory with three or more compatibility conditions. We have carried out intensive numerical calculation and found that:

(i) Shock ray theory gives results which agree very well with Euler solution results compared to the agreement of the results of GSD.

(ii) The results of shock ray theory show that nonlinear waves on the shock front help a non-circular shock to evolve into a circular one quite rapidly.

(iii) We show theoretically that there are limitations on the applicability of weakly nonlinear ray theory, GSD and shock ray theory to a piston problem when the piston has a corner making an obtuse angle to the flow: the piston may move with a small velocity to produce a weak shock, but its velocity should not be too small when the angle of the wedge is fixed.

(iv) The difference in the solution of GSD and Euler solution may become more significant for a strong shock with larger values of $V$. The shock ray theory for a strong shock has been formulated with only two compatibility conditions (Prasad 2004) and we hope that the new formulation will lead to equations, which also give good results.

The comparison and limitations discussed in this paper are very important. GSD and shock ray theory with kinematical conservation laws are now very powerful theories to solve many practical problems and they also take considerably less time than the Euler solution. As mentioned in $\S 1$, attempts have been made to discuss some finer limiting results of the shape of a shock front by GSD (Apazidis et al. 2002). Such results are acceptable only if it can be shown that the error between the GSD results and the solution (not numerical, but exact) of the Euler's equations are much smaller than those involved in the finer structures. What we see from the comparison of the results is that GSD will certainly fail in reproducing almost all limiting results. We may use shock ray theory with caution or probably use a higher-order shock ray theory.

The authors express their sincere thanks to Professor G. Warnecke for his advice in the use of the numerical schemes. They thank AR\&DB, Ministry of Defence, Government of India for financial support through the project 'Nonlinear Hyperbolic Waves in Multi-dimensions with Special Reference to Sonic Booms' (no. DARO/08/1031199/M/I) and thank Professor S. Poedt for providing a visiting position to P. P. under project OT/02/57 at Katholiek University Leuven.

\section{REFERENCES}

Apazidis, N., Lesser, M. B., Tillmark, N. \& Johanson, B. 2002 An experimental and theoretical study of converging polygonal shock waves. Shock Waves 12, 39-58.

BASKar, S. \& PRASAD, P. 2004 Riemann problem for kinematical conservation laws and geometrical features of nonlinear wavefront. IMA J. Appl. Maths 69, 391-420.

Cockburn, B., San, Y. L. \& Shu, C. W. 1989 TVB Runge-Kutta local projection discontinuous Galerkin finite element method for conservation laws III: one-dimensional systems. J. Comput. Phys. 84, 90-113.

GrinfEL'D, M. A. 1978 Ray method for calculating the wavefront intensity in nonlinear elastic material. PMM J. Appl. Maths Mech. 42, 958-977.

Kevlahan, N. K. R. 1996 The propagation of weak shocks in non-uniform flow. J. Fluid Mech. 327, 167-197.

Maslov, V. P. 1980 Propagation of shock waves in an isentropic non-viscous gas. J. Sov. Maths 13, 119-163 (Russian publication 1978). 
Monica, A. \& Prasad, P. 2001 Propagation of a curved weak shock front. J. Fluid Mech. 434, $119-151$.

Plotkin, K. J. 2002 State of the art of sonic boom modeling. J. Acoust. Soc. Am. 111, 530-536.

Prasad, P. 1982 Kinematics of a multi-dimensional shock of arbitrary strength in an ideal gas. Acta Mech. 45, 163-176.

Prasad, P. 1993 Propagation of a Curved Shock and Nonlinear Ray Theory. Pitman Research Notes in Mathematics Series, vol. 292. Longman.

PRASAD, P. 1995 Formation and propagation of singularities on a nonlinear wavefront and a shock front. J. Indian Inst. Sci. 75, 517-535.

Prasad, P. 2000 An asymptotic derivation of weakly nonlinear ray theory. Proc. Indian Acad. Sci. Math. Sci. 110, 431-447.

Prasad, P. 2001 Nonlinear Hyperbolic Waves in Multi-Dimensions. Monographs and Surveys in Pure and Applied Mathematics, vol. 121. Chapman \& Hall/CRC.

Prasad, P. 2004 Propagation of a curved shock of arbitrary strength. Preprint, Bangalore: Department of mathematics, Indian Institute of Science.

Prasad, P., Ravindran, R. \& SaU, A. 1991 On the characteristic rule for shocks. Appl. Maths Lett. 4, 5-8.

Prasad, P. \& SANGEETA, K. 1999 Numerical simulation of converging nonlinear wavefronts. J. Fluid Mech. 385, 1-20.

Ravindran, R. \& Prasad, P. 1990 A new theory of shock dynamics, Part I: analytic considerations. Appl. Maths Lett. 3, 77-81.

Schwendeman, D. W. 2002 On converging shock waves of spherical and polyhedral form. J. Fluid Mech. 454, 365-386.

Sturtevant, B. 1989 The physics of shock focusing in the context of extracorporeal shock wave lithotripsy. Proc. Intl Workshop on Shock Wave Focusing (ed. K. Takayama). Shock Wave Research Centre, Institute of Fluid Science, Tohoku University, Tohoku.

Sturtevant, B. \& Kulkarni, V. A. 1976 The focusing of weak shock waves. J. Fluid Mech. 73, 651-671.

Whitham, G. B. 1957 A new approach to shock dynamics. Part 1. Two-dimensional problems. J. Fluid Mech. 2, 146-171.

Whitham, G. B. 1959 Some comments on wave propagation and shock wave structure with application to magnetohydrodynamics. Commun. Pure Appl. Maths 12, 113-158.

Whitham, G. B. 1974 Linear and Nonlinear Waves, John Wiley. 